\title{
Engineering tensile behavior of hybrid carbon fiber/self-reinforced polypropylene composites by bio-inspired fiber discontinuities
}

Jun Tang ${ }^{1 *}$, Yentl Swolfs ${ }^{1}$, Arya Aslani ${ }^{1}$, Lorenzo Mencattelli ${ }^{2}$, Gianmaria Bullegas ${ }^{2}$, Silvestre T. Pinho ${ }^{2}$, Stepan V. Lomov ${ }^{1}$, Larissa Gorbatikh ${ }^{1}$

${ }^{1}$ Department of Materials Engineering, KU Leuven, Kasteelpark Arenberg 44 box 2450, 3001 Leuven, Belgium

${ }^{2}$ Department of Aeronautics, Imperial College London, South Kensington, SW7 2AZ, UK

Abstract: Carbon fiber layer failure is vital for the tensile behavior of interlayer hybrid carbon fiber (CF)/selfreinforced polypropylene (SRPP) composites. Introducing cuts, termed here as discontinuities, into the carbon layer is a promising way to tailor its failure behavior. Inspired by structural features of biological composites, we designed and produced hybrid composites with hierarchical and polygonal arrangements of discontinuities in the carbon layer. Increasing the number of levels in the hierarchical patterns delayed the onset of carbon layer failure, hence improving the damage resistance of the hybrid composites. A progressive carbon layer failure was achieved with the polygonal patterns by creating a transition from fiber bundle pull-out to fiber bundle fracture. Spreading the polygonal patterns throughout the specimen resulted in a unique diffused delamination distribution that has not been reported in the literature. Pseudo-ductile behavior was achieved by creating dispersed fiber bundle pullout with the fully dispersed polygonal patterns. The resulting hybrid CF/SRPP composites demonstrated a rare combination of stiffness (10 GPa) and ductility ( $16 \%$ failure strain) with a pseudo-ductile strain over $14 \%$. This paper delivers and proves the concept of utilizing discontinuities to engineer the tensile behavior of hybrid composites.

Keywords: A. Discontinuous reinforcement; A. Hybrid; B. Delamination; B. Fracture

\section{Introduction}

Traditional carbon fiber-reinforced composites are now widely used thanks to their excellent mechanical properties. However, the extension of their applications is still limited by: (1) the lack of ductility due to the inherent brittleness of carbon fiber, and (2) the lack of damage dispersion capability, i.e. early localization of damage leading to premature failure. To overcome these challenges, hybridizing carbon fiber with a more ductile fiber, such as glass fiber, has been a promising strategy [1,2]. Firstly, these fiber-hybrids withstand strains of their ductile component, which means they have higher ductility than the brittle component only. Secondly, the hybrids can still maintain their structural integrity after the brittle component starts fragmenting, thus increasing damage dispersion capability. Research on carbon/glass hybrid composites [3-5] has shown that a gradual, pseudo-ductile behavior is achieved by delocalizing or diffusing fractures in the carbon layer. This process is influenced by many material parameters, including the carbon layer thickness and carbon/glass ratio, which need to be relatively low. 
Hybridizing carbon fiber with self-reinforced polypropylene (SRPP) leads to even higher ductility, as the ultimate failure strain of SRPP is around $20 \%$. Such hybrid CF/SRPP composites exhibit a rare combination of stiffness and ductility in combination with low density. This rare combination stands out from the stiffnessductility dilemma that conventional composite materials suffer from and these hybrids can outperform many traditional materials, mainly engineering plastics and random fiber polymer composites [6]. The problem of continuous CF/SRPP hybrid composites is that the carbon layer failure remains relatively localized. Normally, the carbon layer only fails once or a few times [7,8]. Moreover, the carbon layer fails in an abrupt way by fiber fracture, which leads to an instantly formed delamination with a significant length. As a result, the hybrid suffers from a sudden loss of load-carrying capacity, which is seen as a sudden stress drop on the stress-strain diagram [7]. To avoid the catastrophic failure of the carbon layer and instead to replace it with diffused fractures over a large area, the microstructure of the carbon layer needs some intelligent engineering.

This engineering is inspired by biological materials that are known for their balanced multi-property performance (such as nacre and bone that are both stiff and tough) and intricate microstructures. Typical features of the microstructures include the presence of structural hierarchy and staggered motifs in the arrangement of the discontinuous stiff constituent embedded in a soft matrix [9-13]. These features contribute to the unique combination of stiffness, strength and toughness. Among these three mechanical properties, toughness is intimately linked to the way the material undergoes permanent deformation and diffuses damage. In general, for a given energy dissipation mechanism, damage that is spread over a larger area leads to higher toughness, as more energy is dissipated. The main toughening mechanisms of biological composites include interface shearing and sliding, pull-out of the stiff constituents, process zone toughening, crack bridging, and crack deflection [14,15]. Recent studies $[16,17]$ confirm that the main toughening mechanism of nacre stems from the sliding of aragonite platelets at the sub-micrometer level. Chintapalli et al. [18] found that the strain hardening at the interface is a critical characteristic in toughening of biological materials, as it promotes progressive sliding of the staggered stiff constituent over the entire structure.

Improved manufacturing techniques, such as laser engraving, have made it possible to mimic the microstructures of biological materials in fiber-reinforced composites. Valashani et al. [19] fabricated a nacre-like glass reinforced polyurethane composite by laser engraving. The nacre-like composite has demonstrated considerable amount of glass tablet sliding, crack deflection and branching, which turns the brittle glass into a ductile and deformable material. Narducci and Pinho [20] used laser engraving to manufacture nacre-like carbon fiber composites, which triggered crack deflection under three-point bending. Bullegas et al. [21,22] engineered 
the microstructure of carbon fiber composites with hierarchically-placed micro-laser cuts that are perpendicular to the fiber direction. The hierarchical microstructure significantly increased the translaminar fracture toughness (up to $214 \%$ in [20] and up to $460 \%$ in [22]) compared to the baseline material. This improvement was due to crack deflection and large amount of fiber bundle pull-outs. Furthermore, Henry and Pimenta [23] manufactured bio-inspired carbon fiber composites with hierarchical brick-and-mortar microstructures through laser-engraving. The brick-and-mortar microstructures had two levels of hierarchies, meaning the brick at higher level hierarchy was composed of brick-and-mortar microstructure with a smaller scale. The presence of discontinuities and hierarchies promoted damage diffusion throughout the specimen and hence increased the damage tolerance of the composites.

Although great efforts have been put into bio-inspired composite research [24-26], it has rarely been applied to fiber hybrids. Our previous work $[6,27]$ has proven that introducing discontinuities into the carbon layer of hybrid CF/SRPP composite successfully limited the stress drop caused by carbon layer failure and triggered carbon layer fragmentation. Mencattelli et al. [28] showed that bio-inspired laser cuts can be used to enhance the energy dissipation capability and impact damage tolerance of hybrid CF/SRPP hybrid composites. Although hybrid CF/SRPP composites have demonstrated a rare combination of stiffness and ductility, their tensile behavior is not optimal yet as they suffer from sudden stress drops when carbon layers fail. Moreover, those carbon layer failures are relatively localized, thus limiting the energy dissipation capacity. In the current work, laser cuts with bio-inspired patterns are introduced into the carbon layer of hybrid CF/SRPP composite to optimize the tensile behavior. The aim is to (1) eliminate the stress-drops on the stress-strain curve when the carbon layer fails, hence avoiding the sudden loss of load-carrying capacity, (2) achieve a relatively high stress level when the carbon layer fails and (3) achieve a high ductility. The resultant hybrid CF/SRPP composites with bio-inspired composites should demonstrate a pseudo-ductile behavior with a rare combination of stiffness and ductility.

\section{Materials and methods}

\subsection{Materials}

The materials used in the current study included: 1) woven polypropylene (PP) tape fabrics, 2) PP films, both supplied by Propex Fabrics GmbH (Gronau, Germany), and 3) unidirectional carbon fiber preform, sourced from Chomarat (Le Cheylard, France). The PP tape fabric had an overfed twill $2 / 2$ weave pattern. This means about half of the PP tapes were folded due to the PP tape being wider than the spacing between two adjacent PP tapes [29]. The areal density of the fabric was $130 \mathrm{~g} / \mathrm{m}^{2}$ and the density of PP grade was $0.92 \mathrm{~g} / \mathrm{cm}^{3}$. The compacted thickness of the PP tape fabric was around $141 \mu \mathrm{m}$. The PP film was $20 \mu \mathrm{m}$ thick and was composed of the same PP grade as the tapes, which ensured good compatibility with the PP tapes. The carbon fiber preform had an areal 
density of $71.5 \mathrm{~g} / \mathrm{m}^{2}$. The fiber type was T700SC, with a density of $1.80 \mathrm{~g} / \mathrm{cm}^{3}$. The equivalent thickness of the dry carbon fiber preform was around $40 \mu \mathrm{m}$. The carbon fiber preform was customized by the manufacturer while the coupling agent was confidential.

\subsection{Composite manufacturing}

The fabrication of the hybrid CF/SRPP composites followed three steps: 1) prepregging of the carbon fibers with PP films, 2) laser cutting of the prepregs (perpendicular to the fiber direction) with pre-designed patterns, and 3) hybridization of the laser-cut $\mathrm{CF} / \mathrm{PP}$ prepreg and SRPP.

Firstly, one layer of carbon fiber preform was inserted in between two PP films (one above and one below) and then the stack was hot pressed at $188^{\circ} \mathrm{C}$ and 5 bar for $5 \mathrm{~min}$ to impregnate the fibers. The compacted thickness of the CF/PP prepreg was around $80 \mu \mathrm{m}$.

After production of the CF/PP prepregs, laser micro-engraving was carried out to introduce discontinuities into the prepregs with designed patterns. The laser machine used was Oxford Lasers Diode Pumped Solid State (DPSS) with a focus point diameter of the laser beam ranging from 10 to $15 \mu \mathrm{m}$. The micro-scale laser beam vaporized the material locally and ensured an accurate laser cut. All the cuts had a nominal width of $1 \mathrm{~mm}$ and were perpendicular to the fiber direction. As shown in Fig. 1, the scanning electron microscope (SEM) image of a laser cut demonstrates a clean cut surface, a constant cut gap of around $15 \mu \mathrm{m}$, and a round neat tip with a diameter of $15 \mu \mathrm{m}$. In the area of the heat-affected zone, PP matrix near the fiber ends was partially removed due to the heat flow during the laser milling.

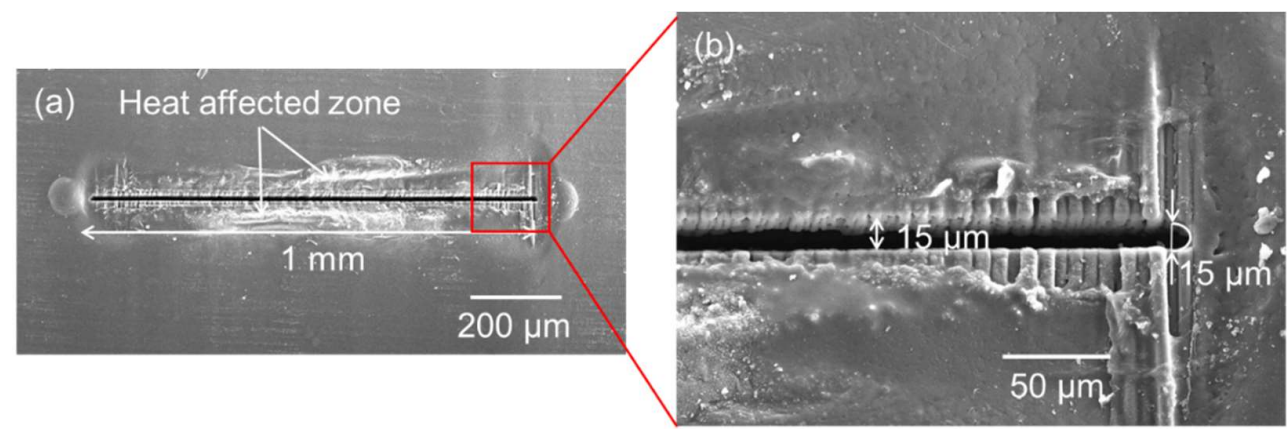

Fig. 1 (a) An SEM image of a $1 \mathrm{~mm}$ laser cut in the CF/PP prepreg, and (b) a zoom-in SEM image of the laser cut showing the heat-affected zone.

In the third step, the hybrid $\mathrm{CF} / \mathrm{SRPP}$ composites were laid up with the stacking sequence $\left[\mathrm{SRPP}_{3} / \mathrm{PP}\right.$ film/CF prepreg/PP film/SRPP 3 , as shown in Fig. 2a. The two additional PP films were inserted between the CF prepreg and SRPP layers for two purposes: 1) to increase the hot compaction quality by providing more matrix [30,31], and 2) to re-impregnate the carbon fibers in the heat-affected zone. The stacked materials were hot compacted at $188^{\circ} \mathrm{C}$ for 5 minutes at 39 bar. 


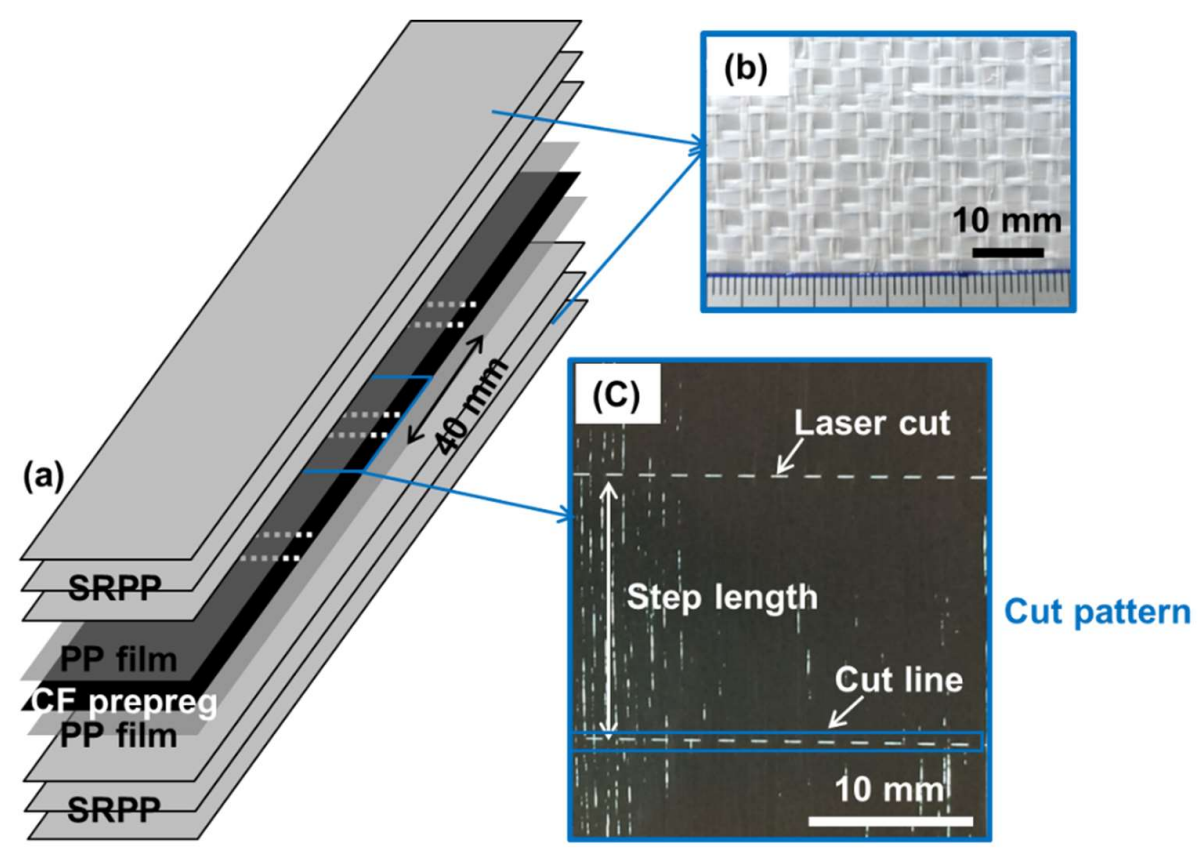

Fig. 2: Schematics showing the structure of hybrid CFPP/SRPP composite with level-1 hierarchical cut pattern: (a) layup of the hybrid, (b) image of the woven PP tape fabric, and (c) image of the cut pattern on the CF/PP prepreg.

During the hot compaction, the outer sheath of the PP tapes was molten to form 'matrix', while the core of the PP tapes remained intact to act as reinforcement. The morphology of the PP tapes after hot compaction can be seen in Fig. 3. A typical volume fraction of the remaining PP tapes after hot compaction was around $70 \%$ in the self-reinforced PP layer [32]. Due to the relatively high pressure applied during the hot compaction, more matrix was squeezed into the cut region. This led to opening of the cut region and consequently forming a resin-rich zone [27]. Those two additional PP films together with the CF prepreg were counted as a whole carbon fiber reinforced polypropylene (CFPP) layer (or carbon layer for simplification). Based on the equivalent thickness of each component in the hybrid composites, the overall carbon fiber volume fraction in the hybrid was calculated to be 4.1\%. The thicknesses and moduli of the components of the hybrids are summarized in Table 1.The adhesion and interlayer bonding is of great importance for the tensile behavior of the hybrid composites [33], but their effect is out of the scope of the current study because all the all the hybrids used the same raw materials and were manufactured with the same processing parameters and the same layup. 


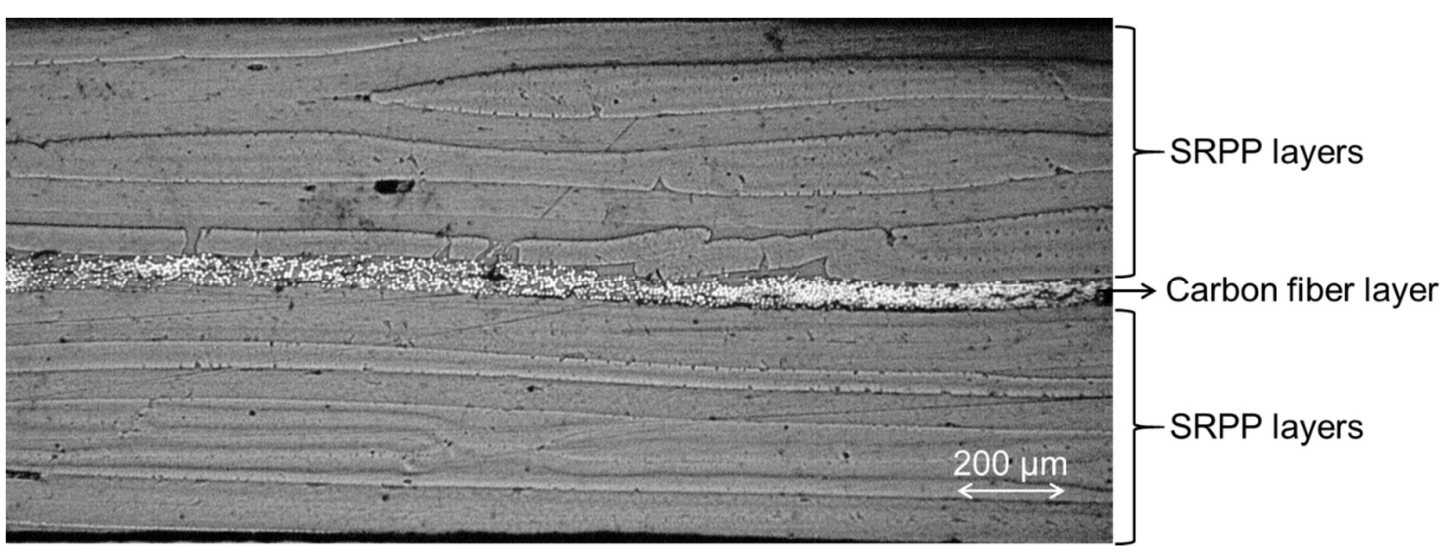

Fig. 3: An optical microscopic image of the cross-section of the hybrid CFPP/SRPP composites showing the morphology of the SRPP.

Table 1: Thicknesses and longitudinal moduli of the components of the hybrid CFPP/SRPP composite.

\begin{tabular}{cccc}
\hline Materials & CFPP without cuts & SRPP & PP film \\
\hline Thickness (mm) & $0.12^{*}$ & $0.14^{*}$ & $0.02^{*}$ \\
Longitudinal modulus (GPa) & $77.7[6]$ & $3.1[6]$ & $1.5[6]$ \\
Strength (MPa) & $1630^{* *}$ & $150^{* *}$ & - \\
\hline
\end{tabular}

* The thicknesses are calculated as areal density over density.

** The strength of the carbon layer without cuts is calculated as the longitudinal modulus multiplied by the failure strain of carbon fibers according to the data sheet (2.1\%). The strength of SRPP is experimental data [29].

\subsection{Pattern design}

In the current study, all the fibers across the specimen width were cut, meaning there were no continuous fibers in the specimen. The adjacent cuts in the fiber direction were always located in a staggered manner. The distance between these two staggered cuts is defined as step length (S), see Fig. 2c. The cut pattern was repeated three times in the longitudinal direction with a distance of $40 \mathrm{~mm}$.

Depending on the shape, the cut patterns were divided into two categories: 1) hierarchical patterns, Fig. 4a, and (2) polygonal patterns, Fig. 4b. The design of cut patterns is inspired by the structural features of biological composites, such as nacre and bone [9-13], including reinforcement discontinuity, staggered and aligned arrangement of the reinforcements. The choice of the pattern parameters are based on the empirical evidence from our previous work [27] and will be compared with the theoretical calculations in Section 3. 


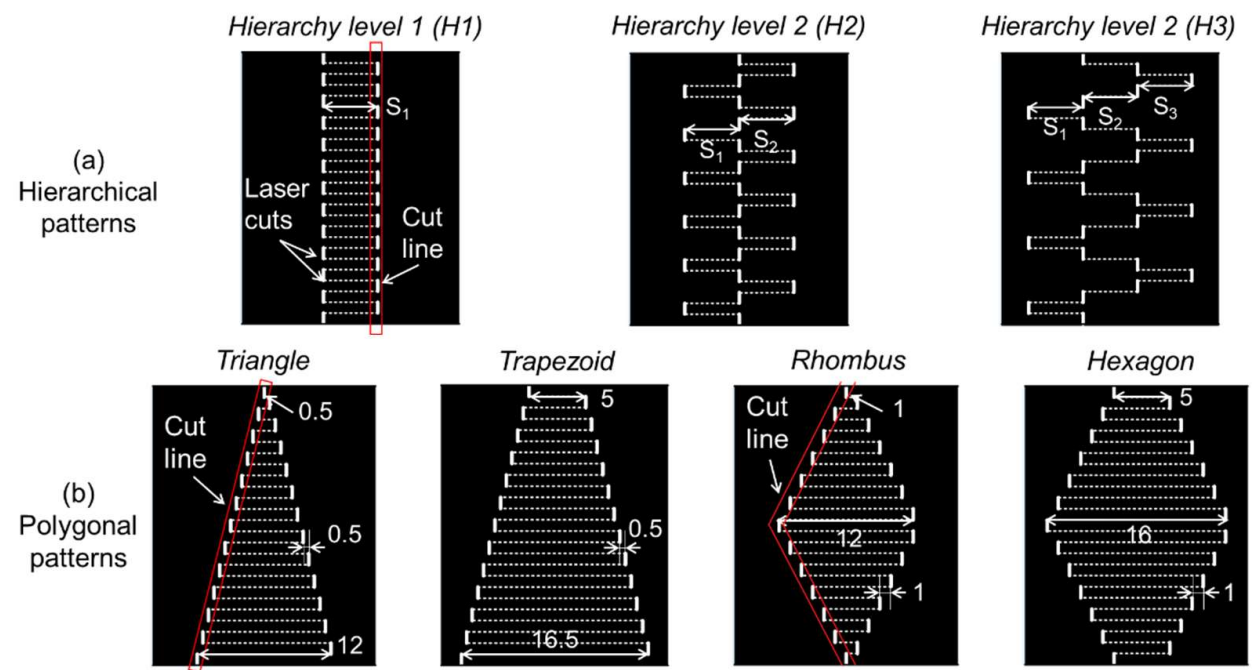

Fig. 4. Schematics of (a) hierarchical patterns and (b) polygonal patterns. The unit of the numbers in (b) is millimeter.

A cut line is composed of a set of laser cuts across the specimen width. The total width of the cuts in the cut line dividing the specimen width is defined as cut density. The $H 1$ pattern is composed of two parallel cut lines that are perpendicular to the fiber direction. Since the two cut lines are parallel to each other, the step length is a constant $\left(\mathrm{S}_{1}\right)$. Splitting one cut line of $H 1$ into two less dense cut lines leads to level-2 hierarchical pattern $(H 2)$. Keeping diluting the cut density by splitting the two cut lines of $H 1$ into four leads to level-3 hierarchical pattern $(H 3)$, see Fig. 4a. The hierarchical structure is built up with an identical step length $\left(\mathrm{S}_{1}=\mathrm{S}_{2}=\mathrm{S}_{3}\right)$ and all the cuts are organized in a staggered manner. Increasing the hierarchy level, on one hand, increases the number of cut lines and decreases the cut density per cut line. On the other hand, higher hierarchy level spreads the cuts more over the entire carbon layer.

By varying the step length of $H 1$, the cut line can be inclined or folded, thus forming the polygonal patterns, see Fig. $4 \mathrm{~b}$. Increasing the step length from a minimum $\left(\mathrm{S}_{\min }\right)$ of $0.5 \mathrm{~mm}$ to a maximum $\left(\mathrm{S}_{\max }\right)$ of $12 \mathrm{~mm}$ with an increment of $0.5 \mathrm{~mm}$ leads to the Triangle pattern. By separating the two inclined cut lines of Triangle pattern to a $\mathrm{S}_{\min }$ of $5 \mathrm{~mm}$, the Trapezoid pattern is generated. If the step length goes up from $1 \mathrm{~mm}$ to $12 \mathrm{~mm}$ and then drops back to $1 \mathrm{~mm}$ with an increment of $1 \mathrm{~mm}$, thus the Rhombus pattern. Increasing the $\mathrm{S}_{\min }$ of Rhombus pattern to 5 $\mathrm{mm}$ yields the Hexagon pattern. The polygonal patterns were first repeated three times along the fiber direction with a distance of $40 \mathrm{~mm}$. In this case, the discontinuities only function at these three specific locations. To allow the discontinuities to function throughout the entire hybrid specimen, two additional patterns were designed, which duplicated the Trapezoid pattern and Hexagon pattern seven times along the specimen, see Fig. 5. They were termed as Trapezoid full and Hexagon full, respectively. As for these two patterns, the closest distance between two adjacent cut patterns was identical to the minimal step length in the cut pattern. 


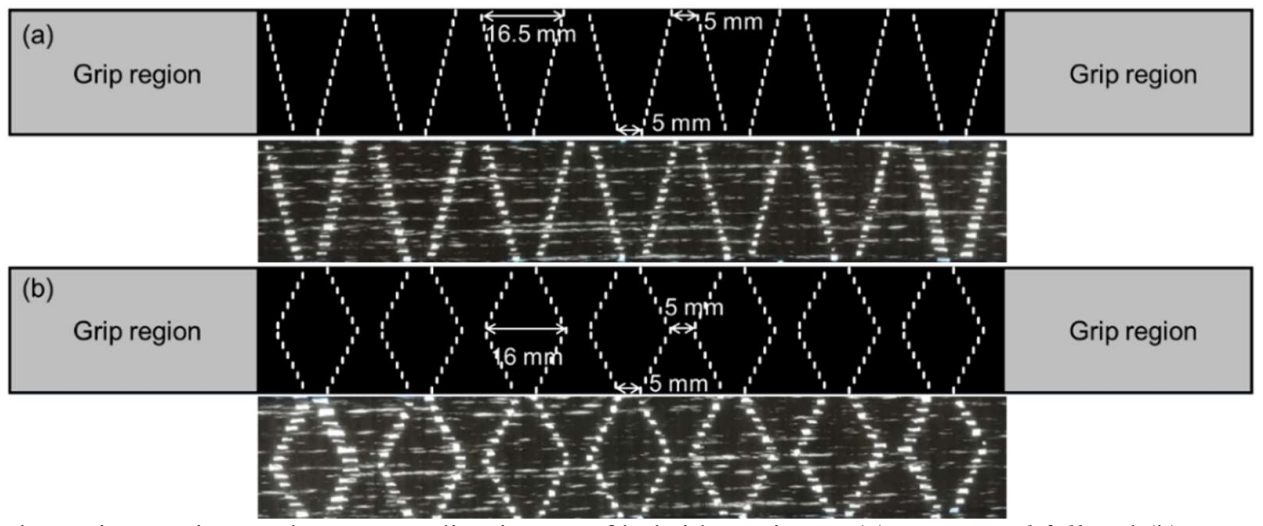

Fig. 5. Schematic top view and corresponding image of hybrid specimen: (a) Trapezoid full and (b) Hexagon full. The image of the specimen demonstrates the opening of laser cuts.

All the tested hierarchical and polygonal patterns are listed in Table 2. To have a better comparison, the baseline specimen, hybrid CF/SRPP composite without laser cuts was also tested.

Table 2: Parameters of the cut patterns

\begin{tabular}{ccccc|ccccc}
\hline $\begin{array}{c}\text { Hierarchical } \\
\text { patterns }\end{array}$ & $\begin{array}{c}\mathrm{S}_{1} \\
(\mathrm{~mm})\end{array}$ & $\begin{array}{c}\mathrm{S}_{2} \\
(\mathrm{~mm})\end{array}$ & $\begin{array}{c}\mathrm{S}_{3} \\
(\mathrm{~mm})\end{array}$ & $\begin{array}{c}\text { Repeated } \\
\text { times }\end{array}$ & $\begin{array}{c}\text { Polygonal } \\
\text { patterns }\end{array}$ & $\begin{array}{c}\mathrm{S}_{\min } \\
(\mathrm{mm})\end{array}$ & $\begin{array}{c}\mathrm{S}_{\max } \\
(\mathrm{mm})\end{array}$ & $\begin{array}{c}\text { Step } \\
\text { increment } \\
(\mathrm{mm})\end{array}$ & $\begin{array}{c}\text { Repeated } \\
\text { times }\end{array}$ \\
\hline$S 5-H 1$ & 5 & & & 3 & Triangle & 0.5 & 12 & 0.5 & 3 \\
$S 5-H 2$ & 5 & 5 & & 3 & Trapezoid & 5 & 16.5 & 0.5 & 3 \\
$S 5-H 3$ & 5 & 5 & 5 & 3 & Trapezoid-full & 5 & 16.5 & 0.5 & 7 \\
$S 10-H 1$ & 10 & & & 3 & Rhombus & 1 & 12 & 1 & 3 \\
$S 10-H 2$ & 10 & 10 & & 3 & Hexagon & 5 & 16 & 1 & 3 \\
$S 10-H 3$ & 10 & 10 & 10 & 3 & Hexagon-full & 5 & 16 & 1 & 7 \\
\hline
\end{tabular}

\subsection{Tensile tests}

The tensile tests were carried out according to the ASTM D3039 standard. The specimens were cut by a hydraulic guillotine and had nominal dimensions of $250 \mathrm{~mm} \times 25 \mathrm{~mm} \times 1 \mathrm{~mm}$ (length $\times$ width $\times$ thickness). They were tested at a displacement rate of $7.5 \mathrm{~mm} / \mathrm{min}$ with a gauge length of $150 \mathrm{~mm}$ ( $5 \%$ strain per min). Sandpaper was used in the gripping region to avoid slippage during the test. Five specimens were tested for each hybrid configuration. Half of the specimen surface, meaning full length times half width, was speckled for strain measurement by 2-dimensional digital image correlation (DIC), while leaving the other half of specimen surface uncoated for direct observation of delamination. The average strain over the entire speckled area was calculated by DIC and used as the tensile strain.

The modulus was calculated as the slope between $0.1 \%$ and $0.3 \%$ strain. The strength was defined as the maximum stress reached. The ultimate failure strain was defined as the strain when SRPP layers fractured. The onset of carbon layer failure reduces the overall stiffness of the specimen, hence causing a deviation from linearity in the stress-strain diagram. The deviation is present on the stress-strain diagram either as a sudden stress drop or a knee point depending on the failure mechanism of the carbon layer. The stress and strain at onset of carbon layer 
failure were obtained from the first peak point on the stress-strain diagram when there was a sudden stress drop, while they were calculated at the intersection point of $0.2 \%$ offset strain when there was a knee point.

\section{Results and discussion}

The permanent deformation in interlayer hybrid composites starts accumulating by 1) failure of the low strain layers (carbon layer in this study); 2) interlaminar delamination between the low and high strain (SRPP in this study) layers; and 3) failure of the high strain layers. The failure of the low strain layers significantly reduces the overall stiffness of the specimen and creates interlaminar delaminations between the low and high strain layers. The delamination propagation affects the ductility of the specimen. The further the delamination propagates, the higher the ultimate failure strain is. This is because the delamination propagation releases the damage localization and allows the high strain layers to elongate more [6,27]. The final failure of the hybrid specimen is due to the failure of the high strain material.

Since the discontinuities were introduced into the carbon layer, they intimately affected the behavior of the carbon layer. The current work focuses on how different patterns influence carbon layer failure behavior. The delamination as well as the failure of SRPP will not be discussed in detail. More information on these failure mechanisms can be found in $[6,24,25]$. The link between the damage in the carbon layer and the overall tensile behavior will be discussed when the stress-strain diagram is present, such as in Fig. 8a and 9a.

\subsection{Hybrids with hierarchical patterns}

\subsubsection{Carbon layer failure}

As shown in Fig. 6a and b, when the hierarchical bundle structure (H3) is loaded in tension, the fiber bundle ends are subjected to shear stress and the stress in the fiber bundle is recovered from zero to the applied stress over the ineffective length [34]. At the same time, the large deformation of the laser cut region (resin rich zone) leads to strain concentration, hence stress concentration, to the neighboring fiber bundle root. Under an increasing tensile load, these two mechanisms are competing against each other. Thus, if the tensile strength of the fiber bundle is reached before the interlaminar shear strength is reached at the interface, the fiber bundles will fracture along the cut line at the fiber bundle root. In the other case, interfacial debonding and fiber bundle pull-out will occur first. The possible failure mechanisms are shown in Fig. $6 \mathrm{c}$ and d. 

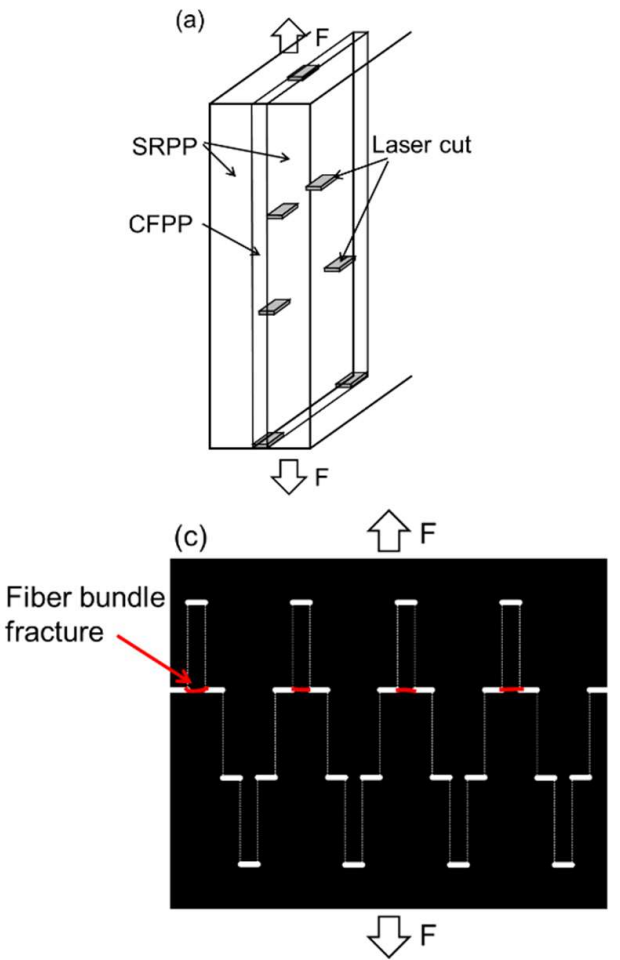

(b)
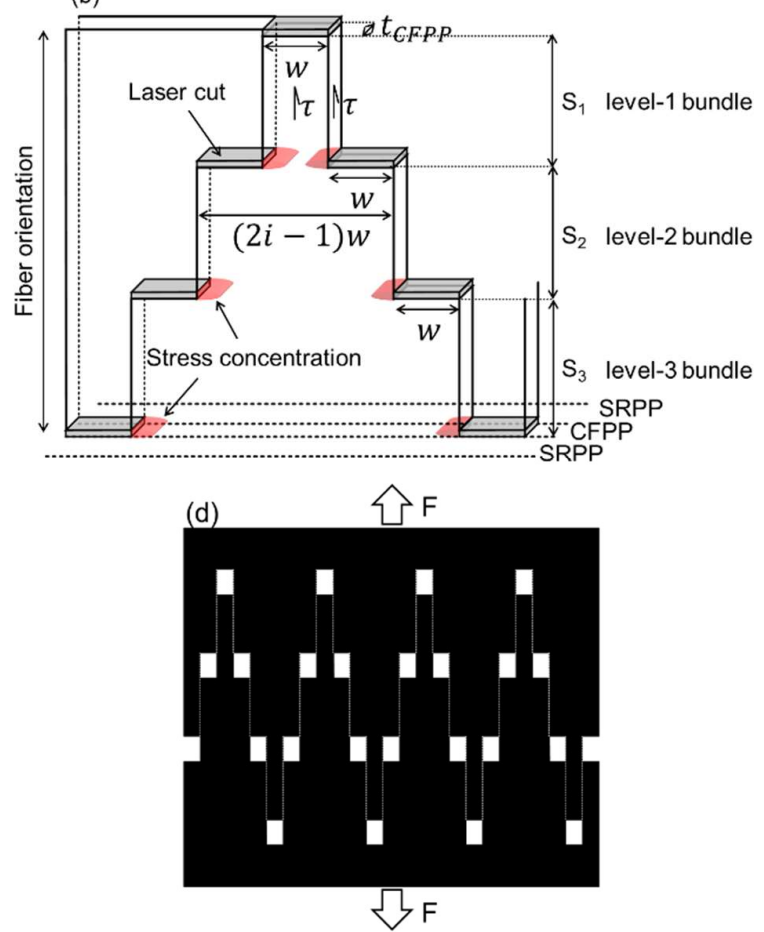

Fig. 6. Schematics of (a) side view and (b) front view of level-3 hierarchical fiber bundle structure under tensile loading, (c) fracture of level-1 fiber bundles, and (d) pull-out of the hierarchical structure.

Bullegas et al. [21] used a probabilistic approach to determine whether the level- $i$ fiber bundle in the hierarchical structure will be pulled out. Here, a simpler deterministic approach to calculate the critical fiber bundle length will be used. Assuming a perfectly-plastic matrix behavior and that there is no fiber bundle debonding yet, the critical length for level- $i$ fiber bundle, $L_{C i}$, should satisfy the following force equilibrium based on the Kelly-Tyson model [35]:

$$
S_{f b} \cdot A_{i}=\tau \cdot\left(\sum_{j=1}^{i-1} C_{j} \cdot L_{j}+C_{i} \cdot L_{C i}\right)
$$

with $L_{j}=\min \left(S_{j}, L_{C j}\right)$.

$\tau$ is the matrix shear strength, which is $10 \mathrm{MPa}$ [36]. $S_{f b}$ is the strength of the fiber bundle. Here, $S_{f b}$ is assumed to be constant for three levels of fiber bundles while ignoring the scaling effect and strength scatter [37]. $S_{f b}$ is calculated as the modulus of the fiber bundle in Table 1 times the failure strain of the carbon fiber $(2.1 \%$ taken from the T700SC datasheet). $A_{i}$ is the cross-sectional area of the fiber bundle and $C_{i}$ is the external perimeter of the level- $i$ fiber bundle. They can be calculated according to Eq. 2 and 3, where $t_{C F P P}$ is the thickness of the carbon layer in Table 1 and $w$ is the width of the laser cut, which is $1 \mathrm{~mm}$.

$$
\begin{gathered}
A_{i}=t_{C F P P} \cdot(2 i-1) \cdot w \\
C_{i}=2 \cdot\left[t_{C F P P}+(2 i-1) \cdot w\right]
\end{gathered}
$$


The item $\sum_{j=1}^{i-1} C_{j} \cdot L_{j}$ is used to account for the contribution of the interlaminar shear stress working on any fiber bundle with level- $j(j<i)$ on the stress build-up in level- $i(i>1)$ fiber bundle. When $i=1$, meaning a single fiber bundle, $\sum_{j=1}^{i-1} C_{j} \cdot L_{j}$ is equal to zero, making Eq. 1 a typical shear-lag equation. $L_{j}$ is the minimal length between the actual length (equal to the step length, $S_{j}$ ) and the critical length of level- $j$ bundle, $L_{C j}$. Thus, if the actual bundle length is higher than the critical length, the critical length should be used because fiber bundle cannot withstand a stress larger than $\frac{\tau \cdot L_{C j} \cdot C_{j}}{A_{j}}$. If the actual length is lower than the critical length, interlaminar shear stress with a value equal to the shear strength $\tau$ acts over the entire fiber bundle, hence the actual length should be used. Here, the assumption is that the stress at the root of the level- $j$ fiber bundle gets immediately redistributed over the whole cross-section of level- $(j+1)$ fiber bundle and the stress gradient in the cross section of level- $(j+$ 1) fiber bundle is neglected.

In the current work, $i=1,2,3$. When the step length is $10 \mathrm{~mm}$, the calculated critical lengths are $8.74 \mathrm{~mm}$, $6.27 \mathrm{~mm}$ and $3.82 \mathrm{~mm}$ for the level-1, -2 and -3 fiber bundle, respectively. Since the critical lengths are all below the actual length, the fiber bundles tend to fracture for S10-Hi patterns. With the increasing of bundle level, the critical length decreases, meaning that the pre-debonding condition is more critical for breaking the higher level fiber bundle. In case of $5 \mathrm{~mm}$ step length, the critical lengths are $8.74 \mathrm{~mm}, 7.62 \mathrm{~mm}$ and $5.42 \mathrm{~mm}$, which are all above the actual bundle length. Hence, the fiber bundles are likely to pull-out for $\mathrm{S} 5$ - $\mathrm{Hi}$ patterns.

The strain concentrations arising from the cuts also play a role in determining the fiber bundle failure. On one hand, they promote fiber bundle pull-out, as they promote the stress recovery in the fiber bundle and hence decreases the critical bundle length. On the other hand, the strain concentrations stimulate the fracture of fiber bundles along the cut line. Globally, with increasing hierarchy level, the number of cuts per cut line is decreased. Since more cuts lead to a larger stiffness reduction of the carbon layer along the cut line, a higher overall strain concentration along the cut line is expected for patterns with a lower hierarchy level. These patterns are therefore expected to fracture earlier. In addition, the cut density of the cut line at the level-1 bundle root is the highest in the $H 1$ and $H 2$ structure and equally highest with that at level-2 bundle root in $H 3$ structure. For the $H 3$ hierarchical structure, the cut line at the level-2 bundle root can be also regarded as the one at the level-1 bundle root of the mirrored pattern. As a result, the level-1 fiber bundles should fracture first in the hierarchical structure. Locally, with an increase of the fiber bundle level in the hierarchical structure, the fiber bundle gets wider and hence the distance between the two cuts next to the fiber bundle root increases, Fig. 6a. This extension reduces the interaction of the two cuts and hence decreases the average strain concentration across the fiber bundle root. This further increases the possibility of triggering fracture of level-1 fiber bundles, as shown in Fig. $6 \mathrm{~b}$. 
The failure mechanisms of the carbon layer were assessed by examining the fracture surface of the specimens, see Fig. 7. In the S5-Hi patterns, the hierarchical fiber bundle structure was maintained well (see Fig. 7a-c), which means the fiber bundles were pulled out. This fits well with the critical length calculations. In $S 10-H i$ patterns, Fig. 7d-f, only level-1 fiber bundles are fractured, which supports the above statement that the level-1 bundles are likely to fracture first in the hierarchical structure. However, according to the analysis above, every level of the fiber bundle should fracture, as the critical lengths for different levels of bundles are all below the step length, 10 $\mathrm{mm}$. The discrepancy is likely due to the fact that when the level-1 fiber bundles fracture, the energy is released in an abrupt way, causing instant formation of an initial delamination [6,27]. The initial delamination partially covers the higher level fiber bundles and the stress in the fiber bundle redistributes along with delamination propagation. The remaining fiber bundle length in the intact region is not long enough for the stress to reach the strength of the fiber bundle. Therefore, the level-2 and level-3 fiber bundle fractures were not found.
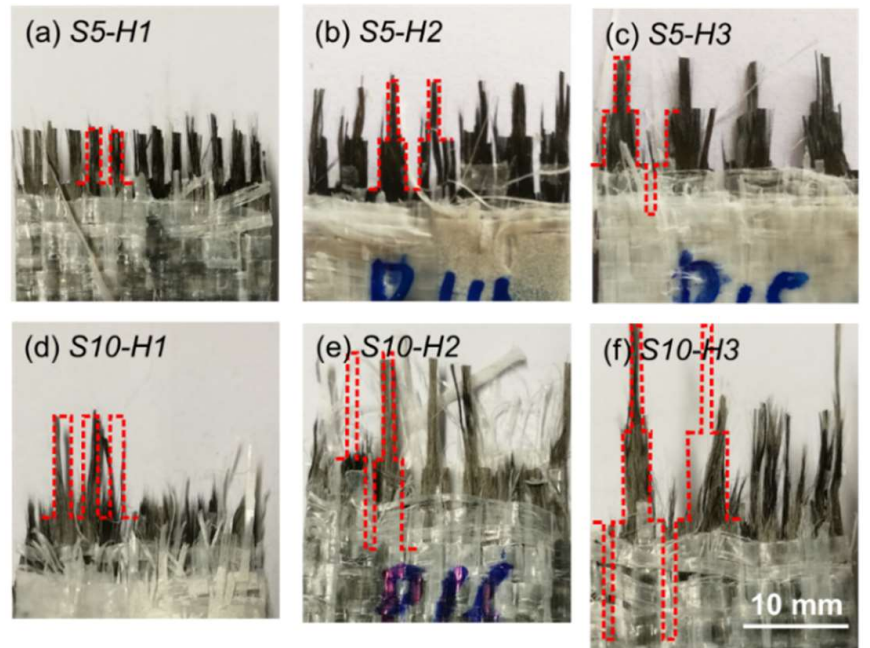

Fig. 7. Images of fracture surface of the hybrid specimens with hierarchical patterns showing (a)-(c) pull-out of hierarchical bundle structure, and (d)-(e) fracture of the level-1 fiber bundles.

\subsubsection{Tensile behavior}

Different mechanisms of carbon layer failure affect differently the stress-strain diagrams. This section describes this link by using $\mathrm{S} 5-\mathrm{H} 2$ and $\mathrm{S} 10-\mathrm{H} 2$ as examples.

Fig. 8 demonstrates a typical stress-strain diagram of an $\mathrm{S} 5-\mathrm{H} 2$ specimen along with the specimen surface images showing the failure process. The whitened region on the images indicates the delamination area between carbon layer and SRPP layer. The whitening in the delaminated region is caused by a change in the way the light passes through the translucent SRPP layers when they delaminate from the carbon layer. As discussed in Section 3.1.1, the carbon layer undergoes bundle pull-out, Fig. 7b. During the pull-out process, the energy is released in a gradual way, hence resulting in a local delamination, see Fig. $8 \mathrm{~b}$. The local delamination decreases the overall stiffness of the hybrid and hence deviates the stress-strain curve from initial linearity, see point b in Fig. 8a. The 
visible delamination initiation in Fig. $8 \mathrm{~b}$ proves that the intersection point of $0.2 \%$ offset strain (point $b$ ) gives a good estimate of the onset of carbon layer failure. Consequently, the same bundle pull-out is observed in the top and middle cut pattern, Fig. 8c and d. This corresponds to the point $\mathrm{c}$ and $\mathrm{d}$ on the stress-strain curve in Fig. 8a. Finally, the hybrid specimen fails by the fracture of SRPP layers, Fig. 8e. As a result, the hybrid partially loses its load-carrying capacity, which is present on the stress-strain curve as a sharp stress drop, see point e in Fig. 8a. Due to the gradual growth of delamination, the stiffness of the specimen decreases smoothly. This leads to a gradual slope change on the stress-strain diagram.

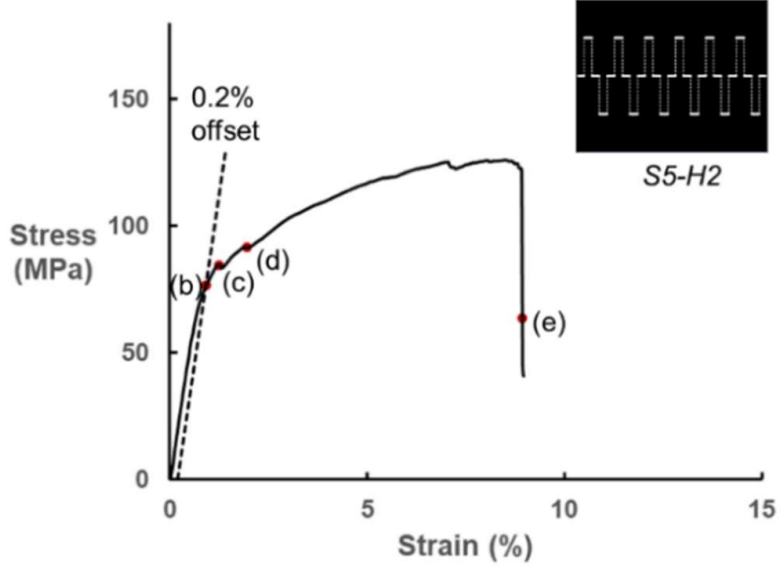

(a)

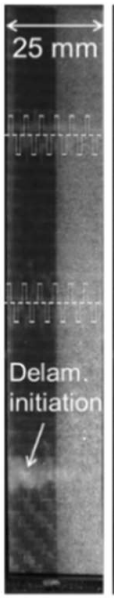

(b)

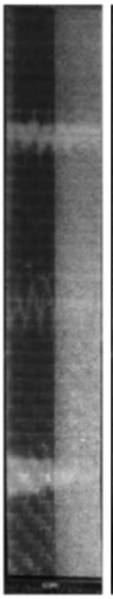

(c)

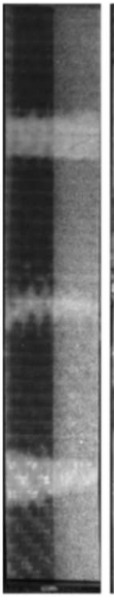

(d)

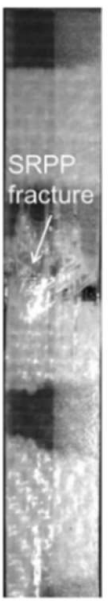

(e)

Fig. 8. (a) A typical stress-strain diagram of $\mathrm{S} 5-\mathrm{H} 2$ specimen experiencing fiber bundle pull-out at three cut patterns. Images of the specimen surface showing (b) clear delamination initiation at the intersection point of $0.2 \%$ offset strain, (c)(d) delaminations initiated by fiber bundle pull-out at the top and middle cut pattern, and (e) final failure of the specimen caused by SRPP layers fracture. The whitening region indicates the delaminated area.

In specimen $\mathrm{S} 10-\mathrm{H} 2$, the carbon layer fails by fiber bundle fracture and when it happens the energy is released in an abrupt way. This results in an initial delamination with a certain length, which is seen on the stress-strain diagram as a sudden stress drop (see Fig. 9a and c). Fig. 9b reveals that there is no apparent delamination observed at the intersection point of $0.2 \%$ offset strain. Hence, in the case of fiber bundle fracture, the stress and strain at the first peak point are used to define the onset of carbon layer failure. Once further loaded, the fiber bundle fracture occurs at the top and middle cut pattern (see Fig. 9d and e). This results in another two stress drops on the stress-strain diagram, see point $d$ and e in Fig. 9a. From point $\mathrm{c}$ to point $\mathrm{d}$ and from point $\mathrm{d}$ to point $\mathrm{e}$, besides the sudden stress drops at point $d$ and e, there is no other stress drop on the stress-strain diagram, meaning no other fiber bundle fracture occurs. This supports the discussion in Section 3.1.1 that only the level-1 bundles are fractured in the hierarchical cut pattern. 


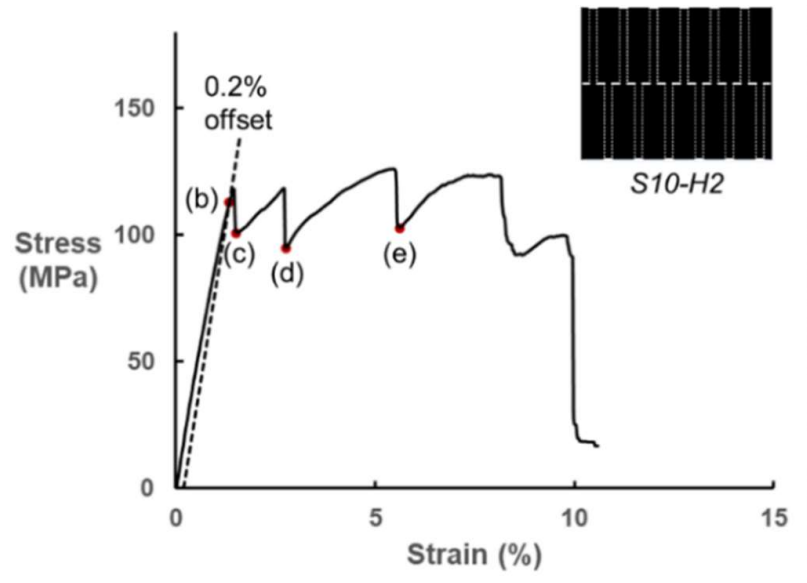

(a)

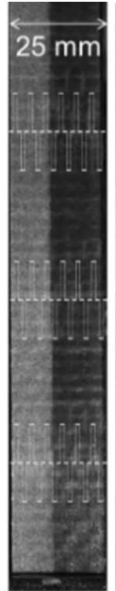

(b)

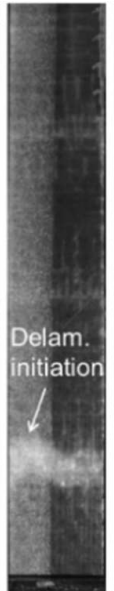

(c)

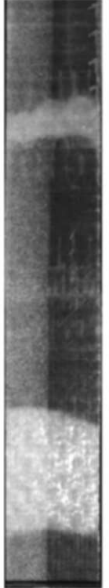

(d)

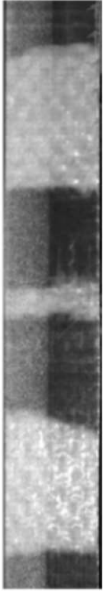

(e)

Fig. 9. (a) A typical stress-strain diagram of $S 10-H 2$ specimen experiencing fiber bundle fracture at three cut patterns. Images of the specimen surface showing (b) no delamination initiation at intersection point of $0.2 \%$ strain offset, (c)(d)(e) delamination initiated by fiber bundle fracture at the bottom, top and middle cut pattern, respectively. The whitening region indicates the delaminated area.

The tensile properties of the hybrids with hierarchical patterns are listed in Table 3 . The representative stressstrain diagrams of the hybrids with hierarchical patterns are plotted in Fig. 10. The baseline hybrid composite is the hybrid CF/SRPP composite with continuous carbon fibers and it has the same layup as the hybrid composite with laser cuts.

Table 3: Tensile properties of the hybrids with hierarchical patterns

\begin{tabular}{cccccc}
\hline $\begin{array}{c}\text { Hierarchical } \\
\text { patterns }\end{array}$ & $\begin{array}{c}\text { Modulus } \\
(\mathrm{GPa})\end{array}$ & $\begin{array}{c}\text { Strain at onset of } \\
\text { carbon layer failure } \\
(\%)\end{array}$ & $\begin{array}{c}\text { Stress at onset of } \\
\text { carbon layer } \\
\text { failure }(\mathrm{MPa})\end{array}$ & $\begin{array}{c}\text { Strength } \\
(\mathrm{MPa})\end{array}$ & $\begin{array}{c}\text { Ultimate failure } \\
\text { strain }(\%)\end{array}$ \\
\hline Baseline & $11.0 \pm 0.4$ & $2.03 \pm 0.05$ & $196.1 \pm 5.7$ & $196.1 \pm 5.7$ & $9.68 \pm 2.37$ \\
S5-H1 & $9.9 \pm 0.6$ & $0.96 \pm 0.04$ & $76.6 \pm 1.4$ & $118.9 \pm 3.8$ & $12.85 \pm 2.84$ \\
S5-H2 & $10.0 \pm 0.6$ & $1.05 \pm 0.09$ & $88.4 \pm 2.3$ & $124.2 \pm 7.1$ & $8.51 \pm 1.34$ \\
S5-H3 & $9.2 \pm 0.3$ & $1.15 \pm 0.07$ & $91.0 \pm 3.8$ & $118.6 \pm 0.7$ & $9.77 \pm 1.74$ \\
S10-H1 & $9.1 \pm 0.4$ & $1.24 \pm 0.05$ & $97.0 \pm 7.4$ & $139.3 \pm 13.5$ & $13.70 \pm 3.83$ \\
S10-H2 & $9.4 \pm 0.3$ & $1.44 \pm 0.05$ & $112.6 \pm 6.1$ & $124.3 \pm 5.3$ & $12.27 \pm 3.07$ \\
S10-H3 & $9.8 \pm 0.7$ & $1.70 \pm 0.08$ & $128.9 \pm 3.5$ & $132.3 \pm 2.2$ & $12.73 \pm 4.24$ \\
\hline
\end{tabular}




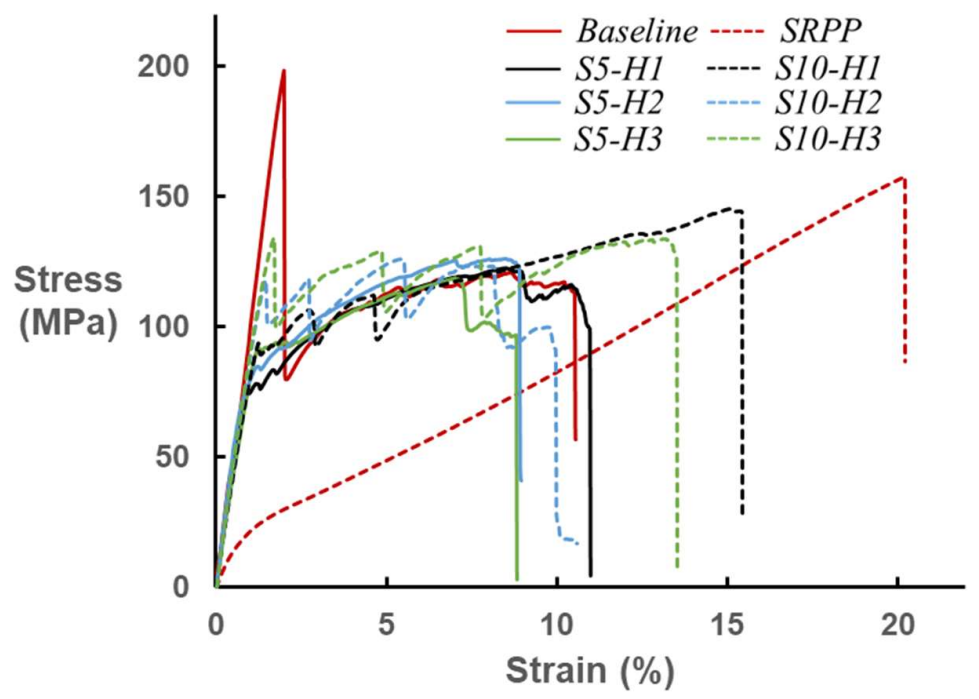

Fig. 10. Stress-strain diagram of hybrid composites with hierarchical cut patterns in comparison with that of the baseline hybrid composite and pure SRPP

Effect of hierarchy level: As shown in Table 3, the strain and stress at onset of carbon layer failure go up with increasing of hierarchy level, irrespective of the step length. This agrees well with the discussion in Section 3.1.1: the cut density and hence the strain concentration along the cut line, is higher for the hybrid with lower hierarchy level. Specimens with lower hierarchy level therefore tend to fail earlier. This also means that increasing the hierarchy level could delay the onset of carbon layer failure.

Effect of step length: The step length affects the failure mechanisms of the fiber bundle. A shorter step length tends to trigger fiber bundle pull-out, which leads to a gradual transition on the stress-strain diagram. A longer step length promotes fracture of the fiber bundle, which results in a sudden stress drop on the stress-strain diagram, see Fig. 10. To eliminate the stress drops on the stress-strain diagram and to achieve a gradual tensile failure behavior, the fiber bundle length should be below the critical bundle length. Comparing the patterns with the same hierarchy level but different step lengths, the stress and strain at onset of carbon layer failure are higher for longer step length, see Table 3. Extending the step length reduces the interaction between the cut lines, which delays the onset of carbon layer failure.

Though the strength and ultimate failure strain are also listed in Table 3, it remains challenging to draw a strong conclusion on these two properties due to the relatively large scatter. The strength and ultimate failure strain are both determined by the failure of SRPP, while the failure of SRPP is a consequence of two competing mechanisms: one is the damage propagation in the SRPP layer, mainly matrix cracks and PP tape debonding, and the other is the stress redistribution caused by delamination propagation [6]. These two mechanisms interact with each other while the specimen is elongating, resulting in the large scatter. 
In comparison with the baseline hybrid composite, the stiffness of the hybrids is slightly reduced due to the presence of laser cuts, see Table 3. The strain and stress at onset of the carbon layer failure are also decreased, which is attributed to the strain concentration caused by the cuts. The advantage of the hybrid composites with hierarchical cut patterns is lowering the stress drop. As shown in Fig. 10, when the continuous carbon fiber layer fractures in the baseline hybrid composite, a large sudden stress drop is present. By introducing laser cuts, the single large stress drops is replaced by multiple small stress drops, hence yielding a more gradual failure behavior. From a strain energy point of view, the energy released upon carbon layer failure is proportional to the square of the applied strain. The released strain energy is mainly dissipated by the formation of delamination. A larger delamination leads to a greater reduction of overall stiffness, hence resulting in a larger stress drop. As shown in Table 3, the strain at onset of carbon layer failure for the baseline hybrid composite is significantly higher than those for the hybrid composite with laser cuts. Therefore, introducing laser cuts actually reduces the strain energy released upon carbon layer failure, hence decreasing the stress drop on the stress-strain curve.

Compared to $3 \mathrm{GPa}$ stiffness of pure SRPP, the resultant hybrid composites have a stiffness around $10 \mathrm{GPa}$. Thus, the stiffness is increased by more than three-fold. The increase in stiffness comes at the cost of a reduced strength and ultimate failure strain. SRPP has a strength around $150 \mathrm{MPa}$ and an ultimate failure strain around $20 \%$, while the strength of the hybrid composites with hierarchical patterns is around 120-140 MPa and the ultimate failure strain is ranging from $8 \%$ to $14 \%$, see Table 3 . With the best hierarchical pattern $(\mathrm{S}=10 \mathrm{~mm})$, the reduction in strength and ultimate failure strain is acceptable. This leads to a more balanced tensile performance.

\subsection{Hybrids with polygonal patterns}

\subsubsection{Carbon layer failure}

For polygonal patterns, the step length (fiber bundle length) varies across the specimen width. The probability of pull-out for shorter bundles is higher than for longer bundles, because the smaller lateral surface of shorter fiber bundle lowers the resistance to bundle pull-out. Therefore, bundle pull-out should initiate from the shortest fiber bundle side, and then propagate towards the longest fiber bundle side. As a result, the delamination between carbon layer and SRPP layer initiated by the bundle pull-out should also propagate from the shortest to the longest fiber bundle side. This can be observed as the whitening region on the specimen surface. If the fiber bundle is long enough, fiber bundle fracture may be triggered. With respect to the critical fiber bundle length calculations in Section 3.1.1, the polygonal pattern can be regarded as level-1 hierarchical pattern but with varied bundle lengths. Since they are working as individual fiber bundles (no hierarchical structure), the critical length for the polygonal pattern is actually equal to that of the level-1 fiber bundle. Based on the calculation, the polygonal cut pattern can be divided into fiber bundle pull-out region and fiber bundle fracture region (see Fig. 11). There might 
be a transition region, as the delamination propagation is a dynamic process and also the fiber bundle strength is not a constant in reality. As shown in Fig. 11, there are fiber bundles that are shorter or longer than the critical fiber bundle length, hence, a mixture of fiber bundle pull-out and bundle fracture are expected for the polygonal patterns. The fractography images of the specimens with polygonal patterns (see Fig. 12) reveal that the carbon layer failed by a combination of these two mechanisms.

(a) Triangle

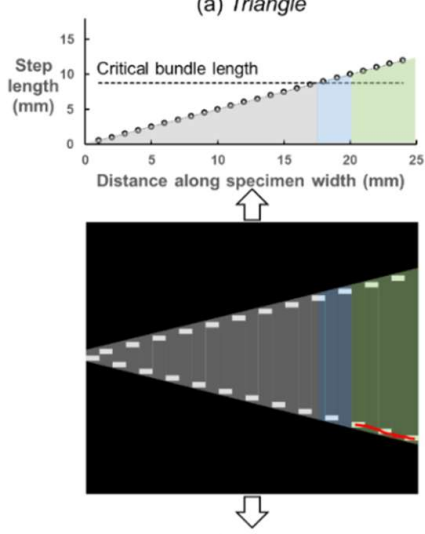

(c) Rhombus
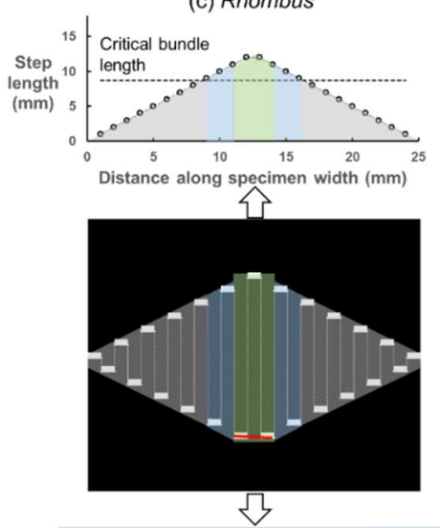

(b) Trapezoid
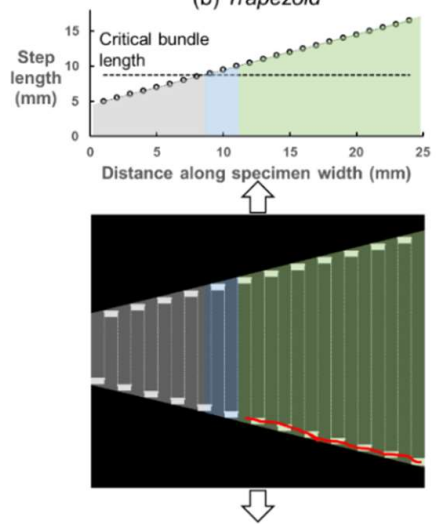

(d) Hexagon
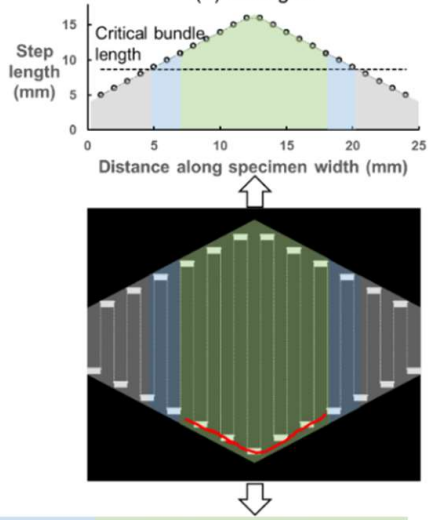

Fiber bundle pull-out Transition zone Fiber bundle fracture

Fig. 11. Predictions of the bundle pull-out region and fracture region in the polygonal cut patterns.

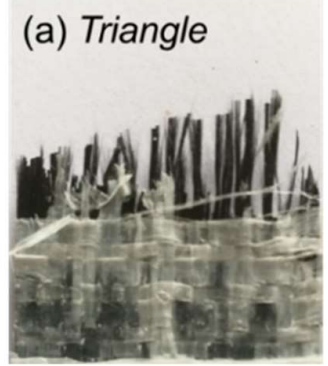

(b) Trapezoid

(c) Rhombus

(d) Hexagon
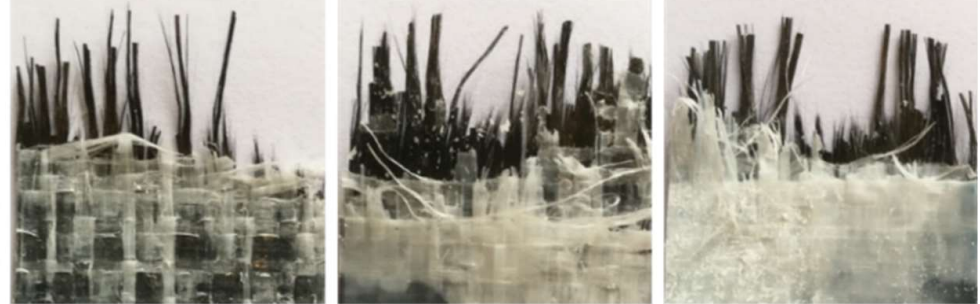

Fig. 12. Images of fracture surface of the hybrid specimens with polygonal patterns showing (a)-(d) a combination of fiber bundle pull-out and fracture.

\subsubsection{Tensile behavior}

Using the Trapezoid specimen as an example, Fig. 13 demonstrates the link between the stress-strain diagram and the failure development. The enlarged images of the bottom pattern of the specimen (see Fig. 13b-e) display 
the process of carbon layer failure in the cut pattern. The delamination is initiated at the shortest fiber bundle side (see Fig. 13b) and propagates gradually towards the longest fiber bundle side (see Fig. 13c). Then, the delamination gets saturated right before the fiber bundle starts fracturing (see Fig. 13d). Finally, the fiber bundle fracture results in an instantly formed delamination along the cut line (see Fig. 13e). Fig. 13f and g reveal the specimen at the moment when the fiber bundle fractures in the middle and top cut pattern. Referring to the stressstrain curve, the delamination initiation induces non-linearity in the stress-strain diagram. The delamination propagation driven by fiber bundle pull-out gradually change the slope of the curve. Then the 'instantly' formed delamination caused by fiber bundle fracture results in a sudden stress drop on the stress-strain diagram. Compared to the stress drops for the specimen with hierarchical patterns, $\mathrm{S1O}-\mathrm{Hi}$, the sudden stress drop here is reduced, as fewer fiber bundles are fractured.

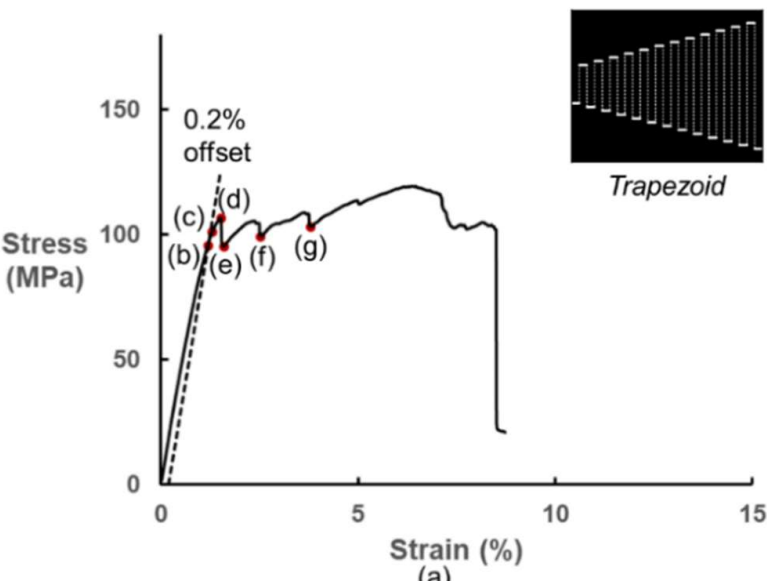

(a)

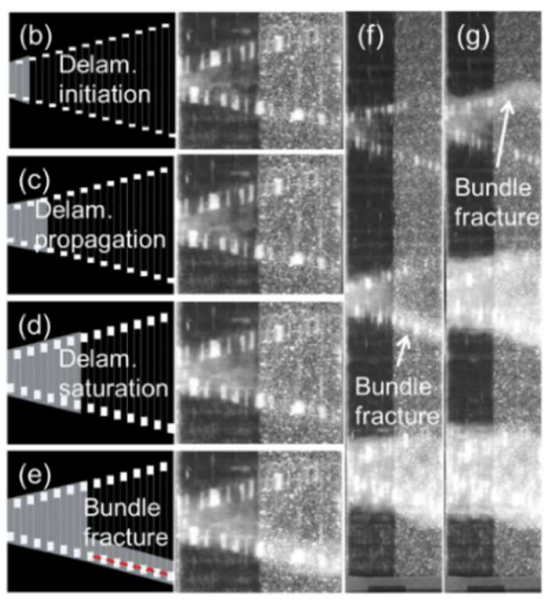

Fig. 13. (a) A typical stress-strain diagram of Trapezoid specimen. Enlarged specimen surface images of the bottom cut pattern showing (b) delamination initiating at the short fiber bundle side, (c) delamination propagating towards long fiber bundle side, (d) delamination saturating in the fiber bundle pull-out region, and (e) delamination caused by fiber bundle fracture. Images of the specimen surface showing delamination when fiber bundle fracture occurs at ( $\mathrm{f}$ ) the middle cut pattern and $(\mathrm{g})$ the top cut pattern. The whitening region indicates the delaminated area.

As for Triangle, Trapezoid, Rhombus and Hexagon specimen, the carbon layer failure remains localized at the three specific cut locations. To achieve a more dispersed carbon layer failure, the cut pattern was repeated as often as possible along the specimen length, as in Trapezoid-full and Hexagon-full, see Fig. 5. 


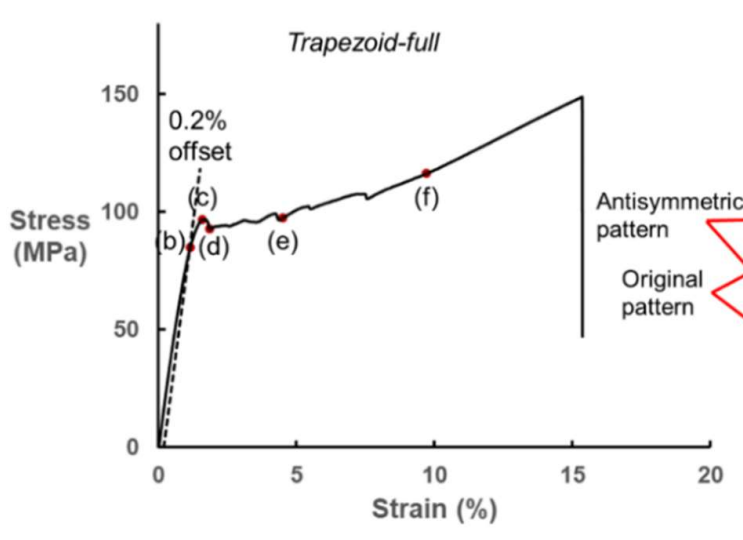

(a)

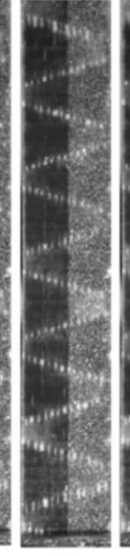

(c)

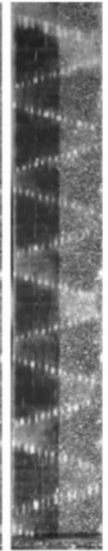

(d)

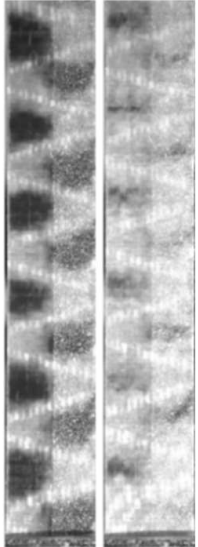

(e)

(f)

Fig. 14. (a) A typical stress-strain diagram of Trapezoid-full specimen. Images of the specimen surface showing (b) delaminations initiating on both sides of the specimen, (c) delaminations propagating towards the long fiber bundle side, (d) delaminations linking up with each other along the cut line, (e) delaminations forming a sineshape distribution, and (f) delaminations merging into a large one. The whitening region indicates the delaminated area.

Fig. 14 shows the failure process along with the stress-strain diagram of Trapezoid-full. The fiber bundle pull-out always initiates from one side of the Trapezoid specimen, while the bundle pull-out in Trapezoid-full can be initiated from both sides of the specimen, see Fig. 14b. This is because the shortest longitudinal distance between two patterns is $5 \mathrm{~mm}$, thus actually resulting in an antisymmetric pattern in between two original patterns. Hence, the pull-out can be started from the shortest fiber bundle of the original pattern and the antisymmetric pattern. The initial delamination area for Trapezoid-full in Fig. 14b is not as visible as for Trapezoid pattern in Fig.13b. This is because there are only three locations for delamination initiation in Trapezoid pattern, while thirteen locations for Trapezoid-full. Since in both cases the image at delamination initiation is taken at the $0.2 \%$ offset intersection point, the total area of the initial delamination should be the same. Therefore, the delamination area per location is much smaller for Trapezoid-full, hence less visible. With the delamination propagation, it becomes more visible (see Fig. 14c). Fig. 14d reveals that the delaminations initiated at opposite sides of the specimen are linking up with each other. Once merged, the delaminations are linked together throughout the specimen, forming a sine-shape distribution (see Fig. 14e). Then, the delaminations keep growing and engulf the entire specimens (see Fig. 14f). By having delaminations initiating on both sides of the specimen and connecting to each other, fiber bundle fracture is avoided. This is attributed to the fact that the delaminations initiated in the antisymmetric pattern release the stress concentration at the long fiber bundles in the original pattern. Similarly, the delaminations created in the original pattern also release the stress concentration at the long fiber bundles in the antisymmetric pattern. As a result, the sudden stress drops on the stress-strain diagram are eliminated, resulting 
in a gradual transition. Also, due to the dispersed fiber bundle pull-outs followed by the delamination propagation throughout the whole specimen, the specimen exhibits a high ultimate failure strain of $16.30 \pm 1.06 \%$.

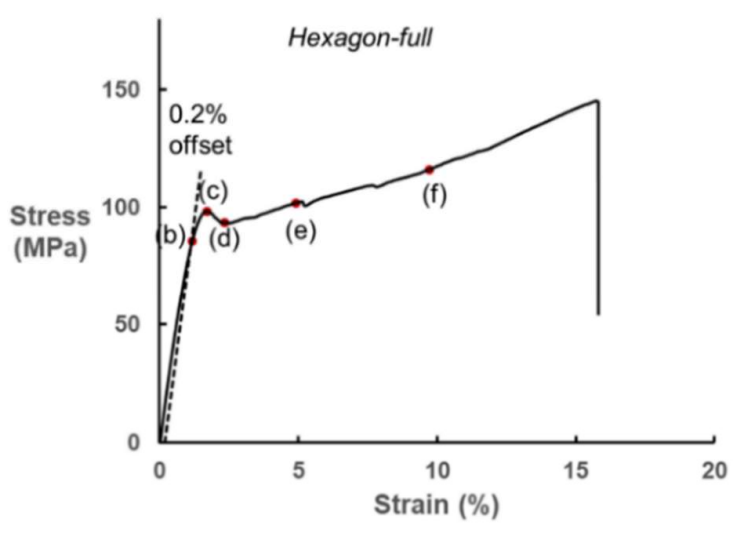

(a)

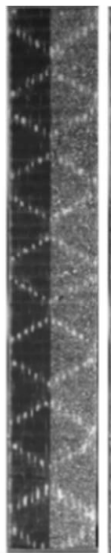

(b)

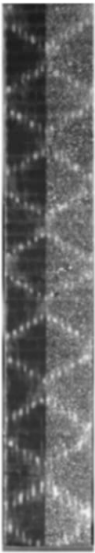

(c)

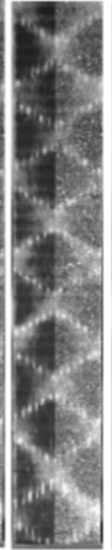

(d)

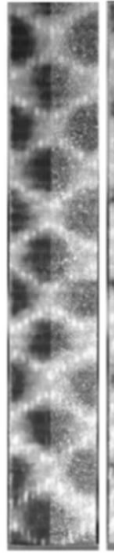

(e)

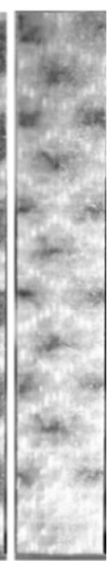

(f)

Fig. 15. (a) A typical stress-strain diagram of Hexagon-full specimen. Images of the specimen surface showing (b) delaminations initiating on both sides and in the middle of the specimen, (c) delaminations propagating towards the long fiber bundle side, (d) delaminations linking up with each other along the cut line, (e) delaminations forming a spread-out distribution, and (f) delaminations merging into a large one. The whitening region indicates the delaminated area.

The stress-strain diagram and the specimen surface images for Hexagon-full are shown in Fig. 15. Comparing to Hexagon pattern, the fiber bundle pull-out can be triggered in the middle of the specimen apart from both sides of the specimen (see Fig. 15b). The reason for the lower visibility of the initial delamination is the same as in Trapezoid-full. The delamination propagation and connection along the cut line results in a spread-out delamination distribution, see Fig. 15d and e. This avoids fiber bundle fracture, thus eliminating the sudden stress drops in the stress-strain diagram. Finally, the dispersed delaminations merge into a large one that covers the entire specimen (see Fig. 15f). Overall, the Hexagon-full shares a similar tensile behavior as Trapezoid-full.

The tensile properties of the hybrids with polygonal patterns are listed in Table 4. The stress-strain diagrams of the hybrids with polygonal patterns as well as the baseline hybrid and pure SRPP are compared in Fig. 16.

Table 4: Tensile properties of the hybrids with polygonal patterns.

\begin{tabular}{cccccc}
\hline $\begin{array}{c}\text { Polygonal } \\
\text { patterns }\end{array}$ & $\begin{array}{c}\text { Modulus } \\
(\mathrm{GPa})\end{array}$ & $\begin{array}{c}\text { Strain at onset of } \\
\text { carbon layer } \\
\text { failure (\%) }\end{array}$ & $\begin{array}{c}\text { Stress at onset of } \\
\text { carbon layer } \\
\text { failure (MPa) }\end{array}$ & $\begin{array}{c}\text { Strength } \\
(\mathrm{MPa})\end{array}$ & $\begin{array}{c}\text { Ultimate failure } \\
\text { strain }(\%)\end{array}$ \\
\hline Triangle & $9.6 \pm 0.2$ & $1.01 \pm 0.03$ & $78.0 \pm 2.0$ & $131.2 \pm 7.2$ & $12.64 \pm 2.67$ \\
Trapezoid & $9.7 \pm 0.2$ & $1.19 \pm 0.05$ & $96.2 \pm 3.7$ & $120.8 \pm 4.3$ & $9.83 \pm 1.56$ \\
Trapezoid-full & $9.1 \pm 0.5$ & $1.12 \pm 0.03$ & $84.1 \pm 3.3$ & $146.4 \pm 7.0$ & $16.30 \pm 1.06$ \\
Rhombus & $9.3 \pm 0.5$ & $1.07 \pm 0.05$ & $80.9 \pm 6.6$ & $113.9 \pm 6.7$ & $8.64 \pm 1.15$ \\
Hexagon & $9.2 \pm 0.2$ & $1.19 \pm 0.04$ & $91.1 \pm 1.9$ & $129.3 \pm 3.6$ & $11.21 \pm 1.40$ \\
Hexagon-full & $8.8 \pm 0.2$ & $1.12 \pm 0.02$ & $81.1 \pm 2.9$ & $147.2 \pm 1.8$ & $15.78 \pm 0.10$ \\
\hline
\end{tabular}




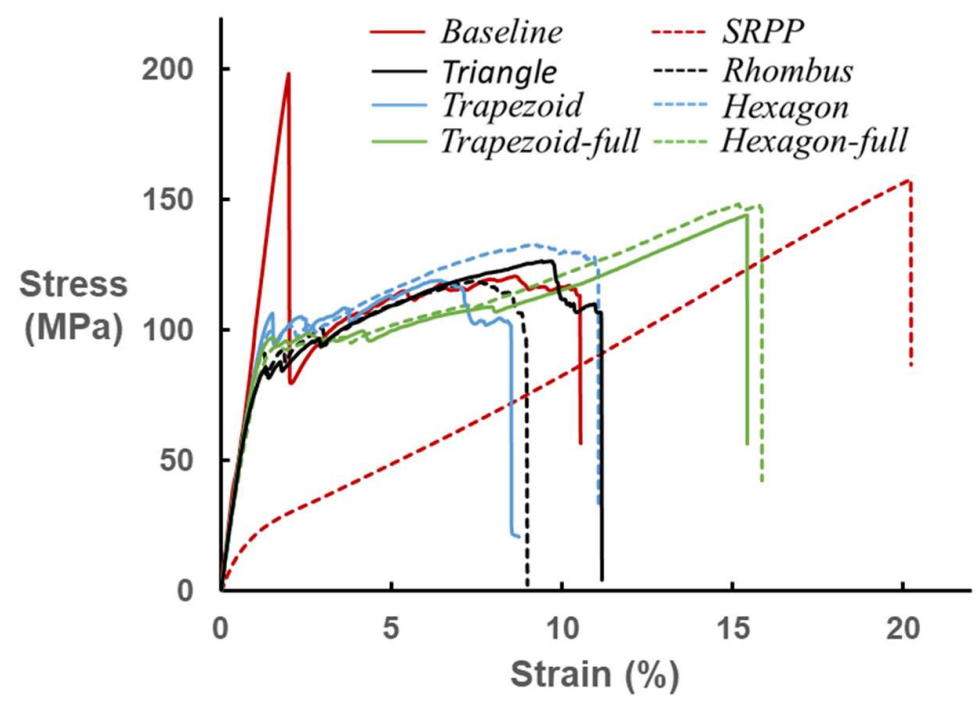

Fig. 16. Stress-strain diagram of hybrid composites with polygonal cut patterns in comparison with that of the baseline hybrid composite and pure SRPP.

Effect of minimum step length: Compared to the Triangle pattern, the minimum and maximum step length of the Trapezoid pattern are larger. The distance between two cut lines in the cut pattern is therefore larger, which decreases the interaction between two cut lines. Hence, the strain concentration should be lower and the onset of carbon layer failure should be delayed. Besides, with a longer minimal step length, the larger lateral surface area of the shortest fiber bundle is increased, which increases the resistance for the fiber bundle to be pulled-out. This further delays the fiber bundle pull-out and hence the onset of carbon layer failure. The data in Table 4 proves that the strain and stress at the onset of carbon layer failure are indeed higher for Trapezoid than for Triangle pattern. The same explanation applies for Rhombus and Hexagon patterns.

Effect of spreading cut pattern throughout the specimen: Comparing Trapezoid and Trapezoid-full patterns, there are more delamination initiators for Trapezoid-full pattern, as spreading the cut pattern over the entire specimen creates more short fiber bundles with the minimal step length. This promotes the onset of carbon layer failure, thereby decreasing the strain and stress at onset of carbon layer failure for Trapezoid-full compared to Trapezoid pattern (see Table 4). In addition, the delamination initiation at the short fiber bundle side of the antisymmetric pattern releases the strain concentration on the long fiber bundles in the original pattern. This phenomenon prevents fiber bundle fracture in Trapezoid-full pattern, resulting in a smooth transition on the stressstrain diagram (see Fig. 16). Moreover, the carbon layer failure is localized at the three cut pattern locations for Trapezoid pattern, while the carbon layer failure is more diffused for Trapezoid-full pattern. More dispersed carbon layer failure leads to a more gradual failure development. Also, the merging of the dispersed delaminations created by the dispersed fiber bundle pull-outs increases the ultimate failure strain, hence the ductility, of the 
specimen, see Table 4. The same trend can be found for Hexagon pattern and Hexagon-full pattern. The stressstrain diagrams of Trapezoid-full and Hexagon-full demonstrate a pseudo-ductile behavior with a large pseudoductile strain, $14.7 \% \pm 1.0 \%$ for Trapezoid-full and $14.1 \% \pm 0.1 \%$ for Hexagon-full.

The tensile properties of the baseline hybrid CF/SRPP composite are given in Table 3. Compared to the baseline hybrid composite, the stiffness of the hybrid composites with polygonal patterns is decreased from 11 GPa to around $10 \mathrm{GPa}$, The stiffness is further reduced to $\sim 9 \mathrm{GPa}$ when spreading the cut patterns over the entire specimen (see modulus for Trapezoid-full and Hexagon-full.in Table 4), as there are more ineffective load-transfer sites. The stress and strain at the onset of carbon layer failure are also reduced due to earlier damage initiation caused by the laser cuts. The strength is reduced a bit, but the stress drop is eliminated. The ultimate failure strain is enhanced when the cut patterns are spread over the entire specimen (see ultimate failure strain for Trapezoidfull and Hexagon-full in Table 4). In this case, the damage is diffused the most and the delamination propagates most extensively, hence yielding the highest ductility.

As shown in Fig. 16, compared to pure SRPP, the stiffness is significantly increased for the hybrid composites with polygonal cut patterns. In terms of Trapezoid-full and Hexagon-full, the ultimate failure strain of SRPP is preserved the most, as it drops from $20 \%$ to $\sim 16 \%$. Meanwhile, a much higher transition stress is obtained thanks to the reinforcing effect of carbon fibers. Therefore, an improved tensile performance is achieved with the Trapezoid-full and Hexagon-full compared to that of pure SRPP.

\section{Benchmarking}

\subsection{Comparison between hierarchical patterns and polygonal patterns}

The carbon layer with hierarchical patterns fails either by pull-out or fracture of the hierarchical bundle structure. The carbon layer failure always occurs along the cut line and across the entire specimen width. When the carbon layer fails by fiber bundle pull-out, a smooth transition is present on the stress-strain diagram, see $S 5$ H1. S5-H2, and S5-H3 in Fig. 10. A higher hierarchy level leads to higher stress and strain at onset of carbon layer failure. When the carbon layer fails by fracture of the hierarchical bundle structure, multiple stress drops appear on the stress-strain diagram, see S10-H1, S10-H2, and S10-H3 in Fig. 10. A higher hierarchy level leads to higher stress and strain at onset of carbon layer failure as well as larger stress drops.

In terms of hybrid CF/SRPP composites with polygonal patterns, the carbon layer fails in a progressive way by damage initiating from the short bundle length side and propagating towards the long bundle length side. A longer initial step length results in a higher yield point. There is not much difference in the yield point between hierarchical patterns and polygonal patterns when the carbon layer fails by fiber bundle pull-out, see the stress and strain at onset of carbon layer failure for $S 5-H 1, S 5-H 2$, and $S 5-H 3$ in Table 3 and those for polygonal patterns 
in Table 4. Instead of fracturing across the specimen width simultaneously in the hybrid composites with hierarchical patterns, the gradual damage development leads to a smoother transition on the stress-strain response, see Fig. 16. However, small stress drops still exist which are caused by the fracture of fiber bundles that are longer than the critical fiber bundle length. Spreading the cut patterns over the entire specimen eliminates these small stress drops by creating widely dispersed fiber bundle pull-out, see Trapezoid-full and Hexagon-full on Fig. 16. Moreover, with these two patterns, the damage is initiated and propagated throughout the entire specimen, which leads to the highest ductility.

In summary, several strategies can be proposed to achieve the optimal tensile performance. To obtain the desired pseudo-ductile behavior, fiber bundle fracture should be avoided to remove the stress drops on the stressstrain diagram. This means that the step length of the cut pattern should be lower than the critical fiber bundle length. To improve the damage resistance, the onset of carbon layer failure should be delayed. A higher hierarchy level or a longer step length would be helpful. To enhance the ultimate failure strain (ductility), the damage should be dispersed as extensive as possible. This can be done by spreading the cut patterns over the entire hybrid composite.

Among all the cut patterns studied in the study, the Trapezoid-full and Hexagon-full patterns are the best thanks to the following reasons: (1) Progressive fiber bundle pull-out over the entire specimen eliminates the stress drops on the stress-strain diagram, thus avoiding the sudden loss of load-carrying capacity. As a result, the pseudoductile behavior is achieved. (2) Spreading the cuts over the entire specimen diffuses the damage most extensively, which results in the highest ductility and toughness.

\subsection{Comparison with aligned discontinuous carbon fiber (ADCF) /SRPP composites}

Besides introducing discontinuities by laser cutting, the discontinuities can also be introduced intrinsically by using aligned discontinuous fibers, as in our previous work [38]. With the best design, both hybrid CF/SRPP composites with polygonal patterns and ADCF/SRPP hybrid composites have demonstrated pseudo-ductile behavior, as shown in Fig. 17. 


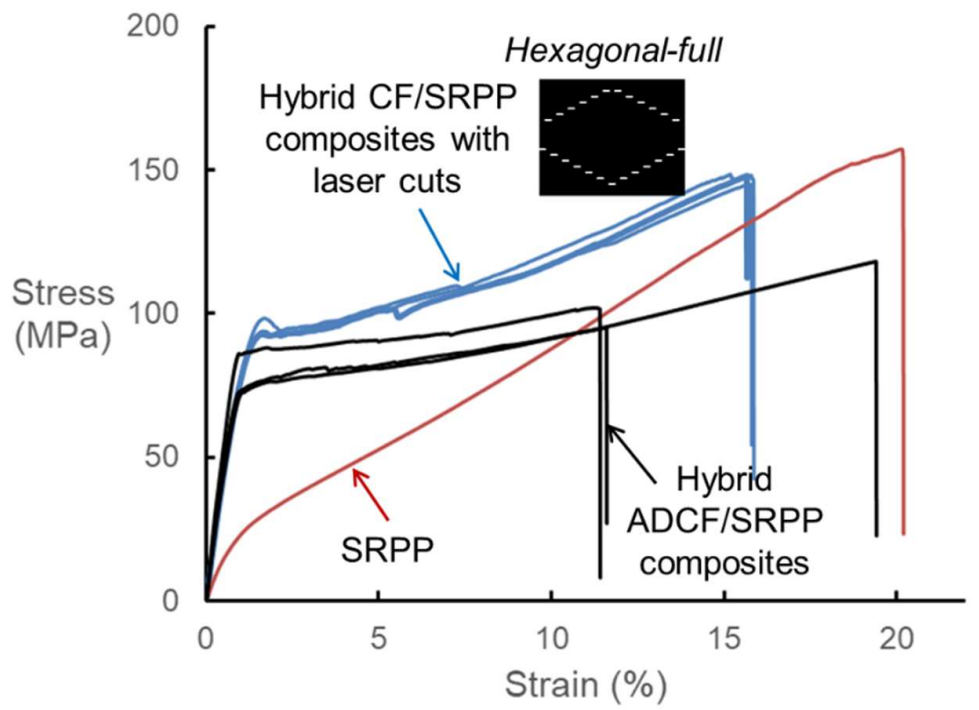

Fig. 17. Stress-strain diagrams of hybrid CF/SRPP composite with hexagonal-full pattern and ADCF/SRPP hybrid composites demonstrating similar pseudo-ductile behavior.

The advantages of using ADCF compared to pre-cut continuous fibers are as follows. Firstly, introducing laser cuts was able to trigger carbon layer fragmentation, but there were still multiple stress drops on the stressstrain curve when the fragmentation was due to fracture of continuous fibers or fiber bundles. The carbon layer in ADCF/SRPP hybrid composites fails by fiber breakage or fiber pull-out, which releases strain energy more gradually and results in a smooth transition in the stress-strain response. Secondly, for the hybrid CF/SRPP composites with laser cuts, the discontinuities were distributed at specific locations, while the discontinuities spread over the entire structure in ADCF/SRPP hybrid composites. This means that the damage can be initiated from anywhere in ADCF/SRPP hybrids, while the damage can be only initiated at the cut sites in CF/SRPP hybrid composites with laser cuts. Therefore, more diffused damage would be expected for the ADCF/SRPP hybrid composites.

The advantage of the laser cutting method is to realize controllable carbon layer behaviour in the non-elastic regime. The damage initiates and propagates following the cut patterns, while the damage initiation and propagation in ADCF/SRPP hybrid composites are random. Therefore, with the laser cutting technique, there is a larger design space for engineers to tailor the tensile performance of hybrid composites. Moreover, the fiber volume fraction in $\mathrm{ADCF} / \mathrm{SRPP}$ hybrid composites was around $30 \%$, while the carbon fiber volume fraction was only around $4 \%$ in the current study. While both hybrids demonstrated a stiffness of around $10 \mathrm{GPa}$, the stiffening efficiency is higher with the laser cutting method. 


\section{Conclusions}

The carbon layer failure is vital for the tensile response of the hybrid CF/SRPP composites. Introducing discontinuities into the carbon layer of the hybrid composites manages to tailor the carbon layer failure by creating fiber bundle pull-out, fiber bundle fracture and a mixture of them. A pseudo-ductile behavior is obtained if the carbon layer fails by fiber bundle pull-out, while there are stress drops on the stress-strain diagram if the carbon layer fails by fiber bundle fracture. More diffused carbon layer failure leads to more ductile tensile behavior.

For hybrid CF/SRPP composites with hierarchical cut patterns, increasing the hierarchy level delays the onset of carbon layer failure, irrespective of the fiber bundle being pulled out or fractured. When the step length (fiber bundle length) is lower than the critical bundle length, the hierarchical bundle structure is pulled out, which results in a gradual transition on the stress-strain diagram. If the step length is higher than the critical bundle length, the fiber bundle fractures, leading to stress drops in the stress-strain diagram. The fiber bundle fracture always happens at the level-1 fiber bundles in the hierarchical structures tested, as strain concentration on the level-1 fiber bundles was the highest.

The carbon layer with polygonal cut patterns failed by a mixture of fiber bundle pull-out and fracture. A progressive carbon layer failure is achieved by bundle pull-out initiating from the shortest fiber bundle side, propagating towards the longest fiber bundle side and bundle fracturing at the long fiber bundle side. Increasing the minimal step length delays the onset of carbon layer failure. Spreading the cut patterns over the entire specimen avoids fiber bundle fracture at the long fiber bundle side, as the strain concentration on the long fiber bundle is

released by the bundle pull-out initiated in between original cut patterns. The diffused carbon layer failure caused by fiber bundle pull-out eliminates the stress drops on the stress-strain diagram and results in a gradual failure with a large pseudo-ductile strain over $14 \%$. Also, the dispersed carbon layer failure leads to a unique diffused delamination distribution following the cut lines, which has never been reported in the literature.

In summary, the current work reveals that a controllable carbon layer failure in interlayer hybrid CF/SRPP composite is achievable by introducing discontinuities with carefully designed patterns. This idea is of importance for engineering the tensile behavior of interlayer hybrid composites. For the future work, the failure behavior of the hybrid CF/SRPP composites with bio-inspired discontinuities under different loading conditions, such as compression, bending, and impact, would be of great interest.

\section{Acknowledgements}

J. Tang would like to acknowledge China Scholarship Council for supporting his PhD study at KU Leuven and the work was partially funded by FWO (Flanders), project G0D5416N. Yentl Swolfs acknowledges FWO Flanders for his postdoctoral fellowship. S.V. Lomov holds the Toray Chair in Composite Materials at KU Leuven, 
the support of which is gratefully acknowledged. Part of the research leading to these results was performed within the framework of the FiBreMoD project and has received funding from the European Union's Horizon 2020 research and innovation programme under the Marie Skłodowska-Curie grant agreement No 722626. S.T. Pinho and G. Bullegas would like to acknowledge funding from EPSRC under grant EP/M002500/1.

\section{References}

[1] Swolfs Y, Gorbatikh L, Verpoest I. Fibre hybridisation in polymer composites: A review. Compos Part A Appl Sci Manuf 2014;67:181-200.

[2] Swolfs Y, Verpoest I, Gorbatikh L. Recent advances in fibre-hybrid composites : materials selection , opportunities and applications. Int Mater Rev 2018:1-35.

[3] Czél G, Rev T, Jalalvand M, Fotouhi M, Longana ML, Nixon-Pearson OJ, Wisnom MR. Pseudo-ductility and reduced notch sensitivity in multi-directional all-carbon/epoxy thin-ply hybrid composites. Compos Part A Appl Sci Manuf 2018;104:151-64.

[4] Czél G, Wisnom MR. Demonstration of pseudo-ductility in high performance glass/epoxy composites by hybridisation with thin-ply carbon prepreg. Compos Part A Appl Sci Manuf 2013;52:23-30.

[5] Jalalvand M, Czél G, Wisnom MR. Damage analysis of pseudo-ductile thin-ply UD hybrid composites A new analytical method. Compos Part A Appl Sci Manuf 2015;69:83-93.

[6] Tang J, Swolfs Y, Yang M, Michielsen K, Ivens J, Lomov SV, Gorbatikh L. Discontinuities as a way to influence the failure mechanisms and tensile performance of hybrid carbon fiber/self-reinforced polypropylene composites. Compos Part A Appl Sci Manuf 2018;107:354-65.

[7] Swolfs Y, Meerten Y, Hine P, Ward I, Verpoest I, Gorbatikh L. Introducing ductility in hybrid carbon fibre/self-reinforced composites through control of the damage mechanisms. Compos Struct 2015;131:259-65.

[8] Swolfs Y, Crauwels L, Breda E Van, Gorbatikh L, Hine P, Ward I, Verpoest I. Tensile behaviour of intralayer hybrid composites of carbon fibre and self-reinforced polypropylene. Compos Part A Appl Sci Manuf 2014;59:78-84.

[9] Eder M, Amini S, Fratzl P. Biological composites - complex structures for functional diversity. Science (80- ) 2018;362:543-7.

[10] Meyers MA, McKittrick J, Chen P-Y. Structural Biological Materials: Critical Mechanics-Materials Connections. Science (80- ) 2013;339:773-80.

[11] Dunlop JWC, Fratzl P. Biological Composites. Annu Rev Mater Res 2010;40:1-24.

[12] Peterlik H, Roschger P, Klaushofer K, Fratzl P. From brittle to ductile fracture of bone. Nat Mater 2006;5:52-5.

[13] Ji B, Gao H. Mechanical Principles of Biological Nanocomposites. Annu Rev Mater Res 2010;40:77-100.

[14] Barthelat F, Rabiei R. Toughness amplification in natural composites. Mater Res Soc Symp Proc 2012;1420:61-6.

[15] Wang R, Gupta HS. Deformation and fracture mechanisms of bone and nacre. Annu Rev Mater Res 2011;41:41-73.

[16] Barthelat F, Tang H, Zavattieri PD, Li CM, Espinosa HD. On the mechanics of mother-of-pearl: A key feature in the material hierarchical structure. J Mech Phys Solids 2007;55:306-37.

[17] Kakisawa H, Sumitomo T. The toughening mechanism of nacre and structural materials inspired by nacre. Sci Technol Adv Mater 2011;12:064710.

[18] Chintapalli RK, Breton S, Dastjerdi AK, Barthelat F. Strain rate hardening: A hidden but critical mechanism for biological composites? Acta Biomater 2014;10:5064-73.

[19] Valashani SM ohamma. M, Barthelat F. A laser-engraved glass duplicating the structure, mechanics and performance of natural nacre. Bioinspir Biomim 2015;10:1-10.

[20] Narducci F, Pinho ST. Exploiting nacre-inspired crack deflection mechanisms in CFRP via microstructural design. Compos Sci Technol 2017;153:178-89.

[21] Bullegas G, Pinho ST, Pimenta S. Engineering the translaminar fracture behaviour of thin-ply composites. Compos Sci Technol 2016;131:110-22.

[22] Bullegas G, Benoliel J, Fenelli PL, Pinho ST, Pimenta S. Towards quasi isotropic laminates with engineered fracture behaviour for industrial applications. Compos Sci Technol 2018;165:290-306.

[23] Henry J, Pimenta S. Increasing damage tolerance in composites using hierarchical brick-and-mortar microstructures. J Mech Phys Solids 2018;118:322-40.

[24] Wegst UGK, Bai H, Saiz E, Tomsia AP, Ritchie RO. Bioinspired structural materials. Nat Mater 2015; 14:23-36.

[25] Studart AR. Towards high-performance bioinspired composites. Adv Mater 2012;24:5024-44. 
[26] Espinosa HD, Rim JE, Barthelat F, Buehler MJ. Merger of structure and material in nacre and bone Perspectives on de novo biomimetic materials. Prog Mater Sci 2009;54:1059-100.

[27] Tang J, Aslani A, Swolfs Y, Bullegas G, Pinho ST, Lomov S V, Gorbatikh L. Staggered ply discontinuities for tailoring the tensile behavior of hybrid carbon fiber/self-reinforced polypropylene composites: A study of pattern parameters. Compos Part A Appl Sci Manuf 2019;125:105551.

[28] Mencattelli L, Tang J, Swolfs Y, Gorbatikh L, Pinho ST. Bio-inspired design for enhanced damage tolerance of self-reinforced polypropylene/carbon fibre polypropylene hybrid composites. Compos Part A Appl Sci Manuf 2019;121:341-52.

[29] Swolfs Y, Crauwels L, Gorbatikh L, Verpoest I. The influence of weave architecture on the mechanical properties of self-reinforced polypropylene. Compos Part A Appl Sci Manuf 2013;53:129-36.

[30] Hine PJ, Olley RH, Ward IM. The use of interleaved films for optimising the production and properties of hot compacted, self reinforced polymer composites. Compos Sci Technol 2008;68:1413-21.

[31] Hine PJ, Unwin AP, Ward IM. The use of an interleaved film for optimising the properties of hot compacted polyethylene single polymer composites. Polymer (Guildf) 2011;52:2891-8.

[32] Jordan ND, Bassett DC, Olley RH, Hine PJ, Ward IM. The hot compaction behaviour of woven oriented polypropylene fibres and tapes. II. Morphology of cloths before and after compaction. Polymer (Guildf) 2003;44:1133-43.

[33] Swolfs Y, Shi J, Meerten Y, Hine P, Ward I, Verpoest I, Gorbatikh L. The importance of bonding in intralayer carbon fibre/self-reinforced polypropylene hybrid composites. Compos Part A Appl Sci Manuf 2015;76:299-308.

[34] Czél G, Jalalvand M, Wisnom MR. Demonstration of pseudo-ductility in unidirectional hybrid composites made of discontinuous carbon/epoxy and continuous glass/epoxy plies. Compos Part A Appl Sci Manuf 2015;72:75-84.

[35] Kelly A, Tyson WR. Tensile properties of fibre-reinforced metals: Copper/tungsten and copper/molybdenum. J Mech Phys Solids 1965;13:329-50.

[36] Burn DT, Harper LT, Johnson M, Warrior NA, Nagel U, Yang L, Thomason J. The usability of recycled carbon fibres in short fibre thermoplastics: interfacial properties. J Mater Sci 2016;51:7699-715.

[37] Pimenta S, Pinho ST. Hierarchical scaling law for the strength of composite fibre bundles. J Mech Phys Solids 2013;61:1337-56.

[38] Tang J, Swolfs Y, Longana ML, Yu H, Wisnom MR, Lomov S V, Gorbatikh L. Hybrid composites of aligned discontinuous carbon fibers and self-reinforced polypropylene under tensile loading. Compos Part A 2019;123:97-107. 